Article

\title{
An Integrated LC-MS-Based Strategy for the Quality Assessment and Discrimination of Three Panax Species
}

\author{
Zhixia Du ${ }^{1,2,+}$, Jinhua $\mathrm{Li}^{2,+}$, Xiang Zhang ${ }^{2}{ }^{\mathbb{D}}$, Jin Pei ${ }^{1, *}$ and Linfang Huang ${ }^{2, *}$ \\ 1 Chengdu University of Traditional Chinese Medicine, Chengdu 611137, China; zxdu712@126.com \\ 2 Key Research Laboratory of Traditional Chinese Medicine Resources Protection, Administration of \\ Traditional Chinese Medicine, National Administration of Traditional Chinese Medicine, Institute of \\ Medicinal Plants, Chinese Academy of Medical Sciences, Beijing 100193, China; pjcdzy03@163.com (J.L.); \\ 18364166427@163.com (X.Z.) \\ * Correspondence: peixjin@163.com (J.P.); lfhuang@implad.ac.cn (L.H.); \\ Tel.: +86-028-61800235 (J.P.); +86-10-5783-3197 (L.H.) \\ + These authors contributed equally to this work.
}

Received: 25 September 2018; Accepted: 6 November 2018; Published: 15 November 2018

\begin{abstract}
The quality assessment and discrimination of Panax herbs are very challenging to perform due to the complexity and variability of their chemical compositions. An integrated strategy was established using UHPLC-Q-Exactive/HRMS and HPLC-ESI-MS/MS to achieve an accurate, rapid, and comprehensive qualitative and quantitative analysis of Panax japonicas (PJ), Panax japonicus var. major (PM), and Panax zingiberensis (PZ). Additionally, discrimination among the three species was explored with partial least squares-discriminant analysis (PLS-DA) and orthogonal partial least squares-discriminant analysis (OPLS-DA) score plots. A total of 101 compounds were plausibly or unambiguously identified, including 82 from PJ, 78 from PM, and 67 from PZ. Among them, 16 representative ginsenosides were further quantified in three herbs. A clear discrimination between the three species was observed through a multivariate statistical analysis on the quantitative data. Nine compounds that allowed for discrimination between PJ, PM, and PZ were discovered. Notably, ginsenoside Rf (G-Rf), ginsenoside F3 (G-F3), and chikusetsu saponin IV (CS-IV) were the three most important differential compounds. The research indicated that the integrated LC-MS-based strategy can be applied for the quality assessment and discrimination of the three Panax herbs.
\end{abstract}

Keywords: qualitative analysis; quantitative analysis; ginsenosides; panax species; UHPLC-QExactive/HRMS

\section{Introduction}

Ginseng herbs, the roots and rhizomes of the Panax species (Araliaceae), are valuable traditional herbs that have a thousand years of medical history and are well-known worldwide as herbal medicines and food to enhance body strength, prevent exhaustion, and improve immunity [1]. Panax species are the important medicinal resources in the world because of their significant medicinal value, and are distributed in more than 35 countries, particularly America, South Korea, Japan, and China. With their popularity all over the world, the attention that Panax has received has increased significantly [2-5]. These herbs contain complex chemical constituents. Ginsenosides are the characteristic and principal components and have been used as an important index in quality assessment and control. The skeleton of aglycones reveals dammarane tetracyclic triterpenoidal saponins and oleanane pentacyclic triterpenoidal saponins (oleanolic acid-type ginsenosides, OAs) in natural ginsensides. Furthermore, dammarane tetracyclic triterpenoidal saponins can be divided into 
protopanaxatiol-type ginsenosides (PPTs) and protopanaxadiol-type ginsenosides (PPDs) according to the study of dammarane structures [6]. Although numerous analytical approaches have been applied for the qualitative and quantitative analysis of ginsenosides, including UV, IR, TLC, HPLC, and UPLC, the analysis of ginsenosides is still a great challenge because of the diversity, similarity, and complexity of their chemical structures [1].

In recent years, some advanced analytical techniques have been rapidly developed for the quality assessment and control of traditional herbs. Q-Exactive Orbitrap High-Resolution Mass Spectrometry (Q-Exactive/HRMS) is a recently developed technique with extremely high resolution, sensitivity, and mass accuracy. It exhibits a stronger power for the scanning and identification of complex compounds than normal mass spectrometry [7]. LC-HRMS is now a well-established technique for the qualitative analysis of chemical compounds in metabolomic studies, and has seen significant progress in recent years [1]. Combining UHPLC with Q-Exactive/HRMS has been increasingly used to screen and identify complex compounds in herbs and food products [8,9]. Furthermore, high-performance liquid chromatography coupled with electrospray ionization tandem mass spectrometry (HPLC-ESI-MS/MS) is also a powerful tool for complex composition analysis for the quantitation and screening of specific chemicals in foods and plants [10-13]. The MS detector can provide information on molecular formula and fragmentation ions. Moreover, the multiple reaction monitoring (MRM) mode is highly specific and sensitive; it is useful for quantifying complex compounds in natural products [14].

Previous studies show that more than 10 species of the genus Panax are available around the world. Among them, Panax ginseng, Panax quinquefolius, and Panax notoginseng are the most recognized species and have been subjected to extensive study $[15,16]$. The other Panax species are less known. Panax japonicas (PJ), Panax japonicus var. major (PM), and Panax zingiberensis (PZ) are three different ginseng herbs with similar bioactivities; they grow in the mid- and low-latitude areas in the Northern hemisphere $[17,18]$. Similar to other Panax species, they contain a high amount of various chemical constituents, such as saponins, polysaccharides, amino acids and microelements, which are believed to contribute to their multiple bioactivities and restorative functions. PJ is widely used as a traditional medicine in China and Japan and has been recorded in the Chinese Pharmacopoeia and the Japanese Pharmacopoeia $[17,19,20]$. PM, which is thought to be a variety of PJ, has also been recorded in the Chinese Pharmacopoeia [18]. PZ is widely used for strengthening the immune response and providing cardiovascular protection in folk medicines of China and Myanmar [21]. At present, these three ginseng herbs are widely incorporated into health products and dietary supplements for their related and similar pharmacological functions. However, they display certain differences in their activities and effects. Currently, there are few studies on the quantitative and qualitative analysis of the chemical compositions and investigations on the differences between these three Panax herbs. Therefore, it is crucial to study their chemical compositions, perform a quality assessment, and investigate their differences.

In the present study, a new, rapid, and sensitive UHPLC-Q-Exactive/HRMS analysis method has been developed for the first time to comprehensively screen and identify the chemical compositions of the three ginseng herbs PJ, PM, and PZ. A sensitive and practical HPLC-ESI-MS/MS method was established to simultaneously separate and accurately determine the 16 major ginsenosides in 50 batches of the three ginseng samples. Finally, discrimination of the three Panax herbs and the important compounds were investigated using the partial least squares-discriminate analysis (PLS-DA) and orthogonal partial least squares-discriminant analysis (OPLS-DA) methods based on quantitative data.

\section{Materials and Methods}

\subsection{Materials and Reagents}

All ginseng samples are listed in Table 1. Sample materials were collected from China and Myanmar from 2015 to 2016. All samples were authenticated by one of the authors, Professor Linfang 
Huang, and were identified as the radices of P. japonicus C. A. Mey (PJ), P. japonicus C. A. Mey. var. major (Burk) C. Y. Wu et K. M. Feng (PM), and P. zingiberensis C. Y. Wu et K. M. Feng (PZ). The voucher specimens have been deposited at the Herbarium of the Chinese Academy of Medical Science and the Peaking Union Medicinal College.

Table 1. The sources of roots and rhizomes of Panax japonicus var. major (PM1-PM20), Panax japonicas (PJ1-PJ20), and Panax zingiberensis (PZ1-PZ10).

\begin{tabular}{|c|c|c|c|}
\hline No. & Sample & Producing Area & Collection Time \\
\hline 1 & PM-1 & Hongyuan county, Sichuan province, China & 2016 \\
\hline 2 & PM-2 & Hongyuan county, Sichuan province, China & 2016 \\
\hline 3 & PM-3 & Yanyuan county, Sichuan province, China & 2015 \\
\hline 4 & PM-4 & Yanyuan county, Sichuan province, China & 2015 \\
\hline 5 & PM-5 & Wenchuan county, Sichuan province, China & 2015 \\
\hline 6 & PM-6 & Wenchuan county, Sichuan province, China & 2015 \\
\hline 7 & PM-7 & Kangding county, Sichuan province, China & 2016 \\
\hline 8 & PM-8 & Kangding county, Sichuan province, China & 2016 \\
\hline 9 & PM-9 & Kangding county, Sichuan province, China & 2016 \\
\hline 10 & PM-10 & Fugong county, Yunnan province, China & 2015 \\
\hline 11 & PM-11 & Fugong county, Yunnan province, China & 2015 \\
\hline 12 & PM-12 & Ludian county, Yunnan province, China & 2015 \\
\hline 13 & PM-13 & Ludian county, Yunnan province, China & 2015 \\
\hline 14 & PM-14 & Ludian county, Yunnan province, China & 2015 \\
\hline 15 & PM-15 & Nyingchi Prefecture, Tibet area, China & 2015 \\
\hline 16 & PM-16 & Nyingchi Prefecture, Tibet area, China & 2015 \\
\hline 17 & PM-17 & Nyingchi Prefecture, Tibet area, China & 2015 \\
\hline 18 & PM-18 & Nyingchi Prefecture, Tibet area, China & 2015 \\
\hline 19 & PM-19 & Bomi county, Tibet area, China & 2015 \\
\hline 20 & PM-20 & Bomi county, Tibet area, China & 2015 \\
\hline 21 & PJ-1 & En'shi city, Hubei province, China & 2016 \\
\hline 22 & PJ-2 & En'shi city, Hubei province, China & 2016 \\
\hline 23 & PJ-3 & En'shi city, Hubei province, China & 2016 \\
\hline 24 & PJ-4 & Xuan'en county, Hubei province, China & 2016 \\
\hline 25 & PJ-5 & Xuan'en county, Hubei province, China & 2016 \\
\hline 26 & PJ-6 & Leshan city, Sichuan province, China & 2015 \\
\hline 27 & PJ-7 & Leshan city, Sichuan province, China & 2015 \\
\hline 28 & PJ-8 & Meishan city, Sichuan province, China & 2015 \\
\hline 29 & PJ-9 & Meishan city, Sichuan province, China & 2015 \\
\hline 30 & PJ-10 & A'ba county, Sichuan province, China & 2015 \\
\hline 31 & PJ-11 & A'ba county, Sichuan province, China & 2015 \\
\hline 32 & $\mathrm{PJ}-12$ & Ya'an City, Sichuan province, China & 2015 \\
\hline 33 & PJ-13 & Taibai county, Shaanxi province, China & 2015 \\
\hline 34 & PJ-14 & Taibai county, Shaanxi province, China & 2015 \\
\hline 35 & PJ-15 & Taibai county, Shaanxi province, China & 2015 \\
\hline 36 & PJ-16 & Taibai county, Shaanxi province, China & 2015 \\
\hline 37 & PJ-17 & Puer city, Yunnan province, China & 2015 \\
\hline 38 & PJ-18 & Puer city, Yunnan province, China & 2015 \\
\hline 39 & PJ-19 & Jinghong city, Yunnan province, China & 2015 \\
\hline 40 & PJ-20 & Wenshan county, Yunnan province, China & 2015 \\
\hline 41 & PZ-1 & Puer city, Yunnan province, China & 2015 \\
\hline 42 & PZ-2 & Puer city, Yunnan province, China & 2015 \\
\hline 43 & PZ-3 & Puer city, Yunnan province, China & 2015 \\
\hline 44 & PZ-4 & Puer city, Yunnan province, China & 2015 \\
\hline 45 & PZ-5 & Puer city, Yunnan province, China & 2015 \\
\hline 46 & PZ-6 & Puer city, Yunnan province, China & 2015 \\
\hline 47 & PZ-7 & Myanmar, Taung-gyi & 2015 \\
\hline 48 & PZ-8 & Myanmar, Taung-gyi & 2015 \\
\hline 49 & PZ-9 & Myanmar, Taung-gyi & 2015 \\
\hline 50 & PZ-10 & Myanmar, Taung-gyi & 2015 \\
\hline
\end{tabular}


The 16 reference standards of the ginsenosides, that is, ginsenoside (G)-Rg1, G-Re, $R b 1, G-R c$, G-Rb2, G-Rb3, G-Rf, G-Rd, G-Rg2, G-Rg3, G-F3, G-Rh1, G-Rh2, G-Ro, notoginsenoside (NG)-R1, and pseudoginsenoside (pseudo G)-F11, were purchased from Chengdu Must Biotechnology Co., Ltd. (Chengdu, Sichuan, China). The four reference standards of the ginsenosides, that is, G-F1, G-F2, protopanoxadiol (PPD), and protopanaxatriol (PPT), were supplied by the National Institute of Control of Pharmaceutical and Biological Products (Beijing, China). Lastly, two reference standards of chikusetsu saponin (CS), that is, CS-IV and CS-IVa, were obtained from Chengdu Chroma-Biotechnology Co., Ltd. (Sichuan, China). The chemical structures of the 16 quantitative ginsenosides are shown in Figure 1. The purities of all reference standards were higher than $98 \%$, as confirmed by HPLC.
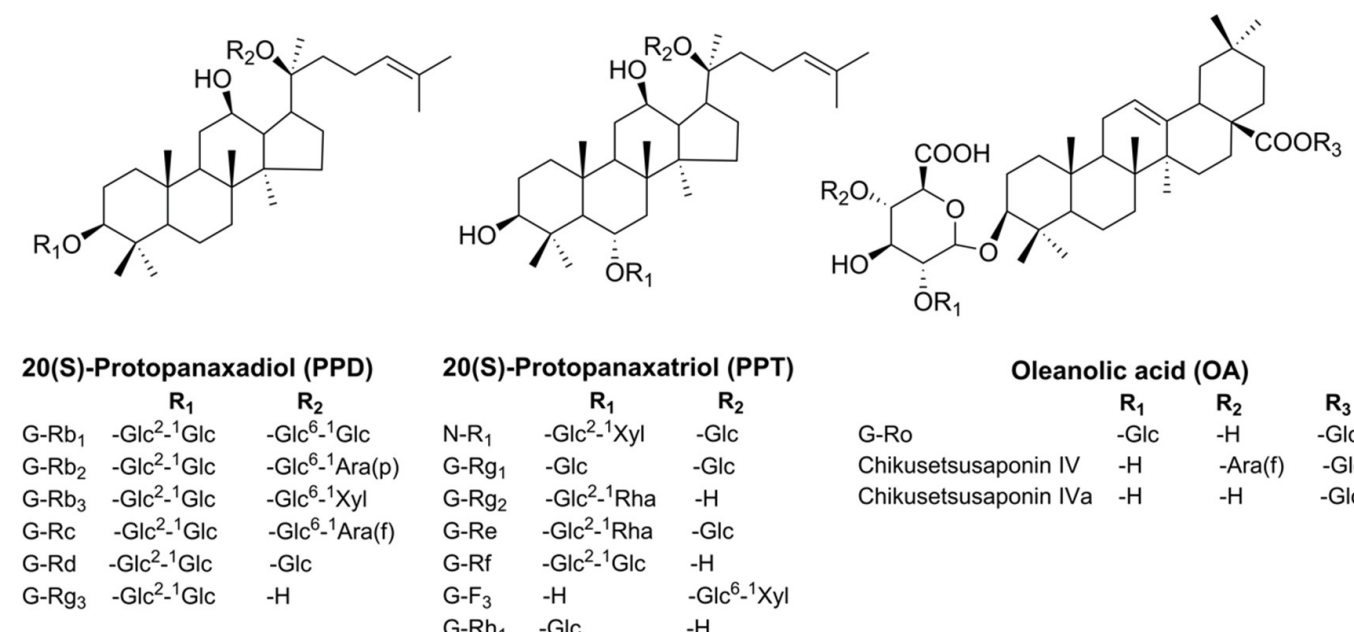

\begin{tabular}{lll}
$\mathbf{2 0}(\mathbf{S})-$ Protopanaxatriol (PPT) \\
\multicolumn{1}{c}{$\mathbf{R}_{1}$} & \multicolumn{1}{c}{$\mathbf{R}_{\mathbf{2}}$} \\
$\mathrm{N}-\mathrm{R}_{1}$ & $-\mathrm{Glc}^{2}-{ }^{-} \mathrm{Xyl}$ & $-\mathrm{Glc}$ \\
$\mathrm{G}-\mathrm{Rg}_{1}$ & $-\mathrm{Glc}$ & $-\mathrm{Glc}$ \\
$\mathrm{G}-\mathrm{Rg}_{2}$ & $-\mathrm{Glc}^{2}-{ }^{-} \mathrm{Rha}$ & $-\mathrm{H}$ \\
$\mathrm{G}-\mathrm{Re}$ & $-\mathrm{Glc}^{2}-{ }^{-} \mathrm{Rha}$ & $-\mathrm{Glc}$ \\
$\mathrm{G}-\mathrm{Rf}$ & $-\mathrm{Glc}^{2}-{ }^{-} \mathrm{Glc}$ & $-\mathrm{H}$ \\
$\mathrm{G}-\mathrm{F}_{3}$ & $-\mathrm{H}$ & $-\mathrm{Glc}^{6}-{ }^{1} \mathrm{Xyl}$ \\
$\mathrm{G}-\mathrm{Rh}_{1}$ & $-\mathrm{Glc}$ & $-\mathrm{H}$
\end{tabular}

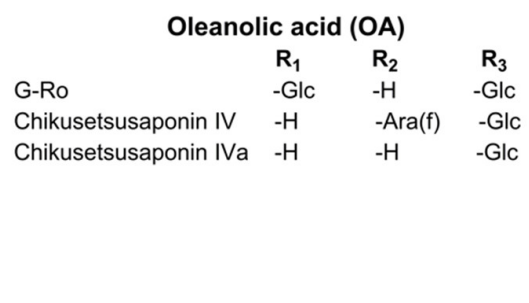

Figure 1. The chemical structures of the 16 reference standards.

LC-MS grade acetonitrile and methanol were obtained from Fisher Scientific (Beijing, China). De-ionized water was purified using a Milli-Q Ultra-pure water system (Millipore, Bedford, MA, USA). All other reagents and chemicals were of analytical grade and obtained from Beijing Chemical Plant Co. Ltd. (Beijing, China).

\subsection{Sample Solutions Preparation}

The samples of PJ, PM, and PZ were pulverized into fine powder in a grinder; $1.0 \mathrm{~g}$ of the powder was suspended in $25 \mathrm{~mL}$ of methanol and ultrasonically extracted $(40 \mathrm{kHz}, 200 \mathrm{~W})$ for $30 \mathrm{~min}$ at $40{ }^{\circ} \mathrm{C}$. The extracted solutions were then filtered through $0.22 \mu \mathrm{m}$ Nylon micropore membranes and used for the UHPLC-Q-Exactive/HRMS analysis.

Furthermore, $0.1 \mathrm{~g}$ of the powder was suspended in $20 \mathrm{~mL}$ of $60 \%$ methanol and ultrasonically extracted $(40 \mathrm{kHz}, 200 \mathrm{~W})$ for $45 \mathrm{~min}$ at $40{ }^{\circ} \mathrm{C}$. The extracted solutions were filtered and diluted 80 times using $60 \%$ methanol. The diluted solutions were filtered through $0.22 \mu \mathrm{m}$ Nylon micropore membranes and used for the HPLC-ESI-MS/MS analysis.

\subsection{Standard Ginsenosides Solutions}

Certain amounts of G-Rg1, G-Re, G-Rb1, G-Rc, G-Rb2, G-Rb3, G-Rf, G-Rd, G-Rg2, G-Rg3, G-F3, G-Rh1, G-Rh2, G-Ro, G-F1, G-F2, PPD, PPT, NG-R1, Pseudo G-F11 CS-IV, and CS-IVa were dissolved in methanol to obtain 22 reference compound stock solutions (at $1.0-5.0 \mathrm{mg} / \mathrm{mL}$ ). The stock solutions were diluted with methanol, and each solution was used for studying fragmentation pathways by a UHPLC-Q-Exactive/HRMS analysis.

Furthermore, a mixed solution containing the references of G-Rg1, G-Re, G-Rb1, G-Rc, G-Rb2, G-Rb3, G-Rf, G-Rd, G-Rg2, G-Rg3, G-F3, G-Rh1, G-Ro, NG-R1, CS-IV, and CS-IVa was also prepared 
and serially diluted with $60 \%$ methanol-water $(v / v)$ to obtain 16 reference solutions with different concentrations, which were used for plotting standard curves in the HPLC-ESI-MS/MS analysis.

\subsection{UHPLC-Q-Exactive Orbitrap HRMS Conditions for Qualitative Analysis}

UHPLC analysis was performed using an Ultimate 3000 system (Dionex, Sunnyvale, CA, USA), which is equipped with an online vacuum degasser, a quaternary pump, an autosampler, and a thermostated column compartment. An ACQUITY UPLC HSS T3, $2.1 \mathrm{~mm} \times 100 \mathrm{~mm}, 1.7 \mu \mathrm{m}$ (Waters, Milford, MA, USA) was used for chromatographic separation at $40{ }^{\circ} \mathrm{C}$. For separation, gradient elution using aqueous formic acid $0.1 \%(v / v)$ was done for mobile phase $\mathrm{A}$ and acetonitrile for phase $B$ at a flow rate of $0.3 \mathrm{~mL} / \mathrm{min}$. The following gradient was applied: $0-1 \mathrm{~min}, 0 \% \mathrm{~B} ; 1-10 \mathrm{~min}$, $0 \% \rightarrow 100 \% \mathrm{~B}$ and $10-10.1 \mathrm{~min}, 0 \% \mathrm{~B}$. The injection volume was $2 \mu \mathrm{L}$, and the injection temperature was set at $15{ }^{\circ} \mathrm{C}$.

High-Resolution Mass spectrometry was performed with a Q-Exactive Orbitrap HRMS (Thermo Fisher, Waltham, MA, USA) using a heated electrospray ionization source (HESI) for the ionization of the target compounds in the negative mode. The operating parameters were as follows: spray voltage, $3.70 \mathrm{KV}$; capillary temperature, $320^{\circ} \mathrm{C}$; sheath gas pressure, $30 \mathrm{psi}$; auxiliary gas pressure, $10 \mathrm{arb}$; auxiliary gas heater temp, $300^{\circ} \mathrm{C}$; scan modes, full MS scan (resolution 70,000); and scan range, $\mathrm{m} / \mathrm{z}$ 100-1500. The data were processed using the Thermo Xcalibur 3.0 software (Thermo Finnigan, San Jose, CA, USA).

\subsection{HPLC-ESI-MS/MS Conditions for Quantitative Analysis}

For quantitative analysis, the separation of the multicomponents was carried out using an Agilent 1260 Infinity liquid chromatography (Agilent, Lexington, MA, USA) equipped with a quaternary pump, an online vacuum degasser, an autosampler, and a thermostatic column compartment. Chromatographic separation was performed on a Waters C18 column $(3.9 \mathrm{~mm} \times 150 \mathrm{~mm}, 4.6 \mu \mathrm{m})$. The mobile phase consisted of (A) acetonitrile and (B) a $0.05 \%$ formic acid aqueous solution by gradient elution (0-3 $\mathrm{min}, 20 \% \rightarrow 23 \%$ A; 3-8 $\mathrm{min}, 30 \% \rightarrow 35 \%$ A; $8-15 \mathrm{~min}, 35 \%$ A; $15-20 \mathrm{~min}, 35 \% \rightarrow 60 \%$ A; $20-22 \mathrm{~min}, 60 \% \rightarrow 80 \%$ A; $22-24 \mathrm{~min}, 80 \% \rightarrow 95 \%$ A; $24-25 \mathrm{~min}, 95 \% \rightarrow 20 \% \mathrm{~A}$ ). The flow rate was $1 \mathrm{~mL} / \mathrm{min}$, and the split ratio was set at 3:2. The temperature was set at room temperature. The injection volume was $10 \mu \mathrm{L}$.

The Applied Biosystems 3200QTRAP triple quadrupole tandem mass spectrometer (Applied Biosystems/MDS Sciex, Concord, Ontario, Canada) used was equipped with an electrospray ionization (ESI) source for the mass analysis and detection. All data collected were analyzed and processed using the Analyst 1.6 software (Applied Biosystems/MDS Sciex. Foster City, CA, USA). The turbo ion spray source was set in the negative ionization mode. Multiple reaction monitoring (MRM) was used for detection transitions. The selective ion-pair, DP, and eV of the 16 saponins are shown in Table 2 . The ion spray voltage was set at $-4500 \mathrm{~V}$, the source temperature was set at $450{ }^{\circ} \mathrm{C}$, and gas 1 and gas 2 were set at 50 psi and 45 psi, respectively.

Table 2. The retention time, precursor ions, product ions, and multiple reaction monitoring (MRM) parameters of the 16 analytes in the negative ion mode. Retention time (TR), declustering potential (DP), apillaryelectrophoresis (CE).

\begin{tabular}{ccccccc}
\hline No. & Analyte & TR $(\mathbf{m i n})$ & Precursor Ion $(\mathbf{m} / \mathbf{z})$ & Product Ion $(\mathbf{m} / \mathbf{z})$ & DP/V & CE/V \\
\hline 1 & NG-R1 & 5.48 & 931.2 & 475.1 & 125 & 70 \\
2 & G-Rg1 & 6.42 & 799.2 & 637.1 & 100 & 55 \\
3 & G-Re & 6.56 & 945.3 & 475.2 & 105 & 62 \\
4 & G-Rf & 11.21 & 799.2 & 475.1 & 100 & 50 \\
5 & G-F3 & 12.09 & 769.2 & 475.1 & 105 & 55 \\
6 & G-Rg2 & 13.14 & 783.2 & 475.1 & 105 & 58 \\
7 & G-Rh1 & 13.27 & 637.2 & 475.1 & 75 & 45 \\
\hline
\end{tabular}


Table 2. Cont.

\begin{tabular}{ccccccc}
\hline No. & Analyte & TR $(\mathbf{m i n})$ & Precursor Ion $(\mathrm{m} / \mathbf{z})$ & Product Ion $(\mathrm{m} / \boldsymbol{z})$ & DP/V & CE/V \\
\hline 8 & G-Rb1 & 13.86 & 1107.2 & 945.1 & 115 & 65 \\
9 & G-Ro & 14.68 & 955.1 & 793.0 & 110 & 60 \\
10 & G-Rc & 14.72 & 1077.2 & 783.2 & 105 & 55 \\
11 & G-Rb2 & 15.70 & 1077.2 & 945.2 & 105 & 50 \\
12 & G-Rb3 & 16.01 & 1077.2 & 783.1 & 100 & 55 \\
13 & CS-IV & 16.31 & 925.1 & 569.1 & 50 & 45 \\
14 & CS-IVa & 17.48 & 793.2 & 569.2 & 35 & 45 \\
15 & G-Rd & 17.51 & 945.2 & 621.2 & 105 & 60 \\
16 & G-Rg3 & 20.68 & 783.2 & 621.2 & 100 & 60 \\
\hline
\end{tabular}

\subsection{Quantitative Method Validation}

To verify linearity, the standard solutions containing 16 reference substances at seven different concentrations were injected into the triple quadrupole tandem mass spectrometer and analyzed. Calibration curves of the reference standards were constructed by plotting the integrated peak area versus the corresponding concentrations. The limit of detection (LOD) and the limit of quantification (LOQ) of the 16 ginsenosides were determined by injecting a series of standard solutions until the basis of response at the signal-to-noise ratio $(\mathrm{S} / \mathrm{N})$ was about 3 times for LOD and 10 times for LOQ. To determine precision, the samples were analyzed six times within the same day. Reproducibility was evaluated by extracting and analyzing six replicates of the same batch of sample with the established method. To determine stability, the same sample solution was analyzed at $0,2,4,8,12$, and $24 \mathrm{~h}$. Furthermore, a recovery test was used to evaluate the accuracy of this method. Known amounts of ginsenoside standards were added into a $0.1 \mathrm{~g}$ of sample six times; the six mixtures were extracted and analyzed.

\subsection{Multivariate Statistical Analysis}

A multivariate statistical analysis was performed using the SIMCA-P 13.0 software (Umetrics AB, Umea, Sweden) using a partial least squares-discriminant analysis (PLS-DA) and an orthogonal partial least squares-discriminant analysis (OPLS-DA). The score plots of all samples employed UV scaling. The heat map was drawn using the Heatmap illustrator 1.0 (Wuhan, Hubei, China).

\section{Results and Discussion}

\subsection{Identity Assignment and Confirmation of the Components in PJ, PM, and PZ}

In this study, the UHPLC-Q-Exactive/HRMS technique was utilized to rapidly separate and comprehensively identify the major compounds in PJ, PM, and PZ. To separate most of the compounds and achieve the most sensitive detection in a short time, the chromatographic and spectral conditions were optimized. Because the negative mode was more sensitive than the positive mode for detecting ginsenosides in the pre-experiment, heated electrospray ionization of the chemical compounds was performed in the negative ion mode. In addition, formic acid, when added to the mobile phase, not only improved the chromatographic peaks, but also easily generated formic acid adductions $[\mathrm{M}+\mathrm{HCOO}]^{-}$, which made it easier to detect and confirm the molecular ion. After optimizing the experimental conditions, the base peak chromatograms were obtained as shown in Figure 2. The details for the identified ginsenosides, such as the retention time (tR), the molecular formula, the theoretical molecular mass, the experimental molecular mass, and MS/MS (fragment ion) information, are summarized in Table 3. These provide abundant information that can be used as a basis for identifying the constituents in the three Panax herbs. The mass error for molecular ions in all identified ginsenosides was within $10 \mathrm{ppm}$, indicating that the experimental molecular formula well-matched with the quasimolecular ions, theoretical molecular ions, and fragment ions. 
Table 3. Characterization of compounds using UPLC-Q-Exactive/MS (PJ, Panax japonicas; PM, Panax japonicus var. major; PZ, Panax zingiberensis).

\begin{tabular}{|c|c|c|c|c|c|c|c|c|}
\hline No. & $\mathrm{T}_{\mathrm{R}} / \mathrm{min}$ & Formula & \multicolumn{2}{|c|}{$[\mathbf{M}-\mathbf{H}]^{-}$} & {$\left[\mathrm{M}+\mathrm{HCOO}^{-}\right.$} & MS/MS & Identification & Sample \\
\hline & & Calculated & Measured & & & & & \\
\hline 1 & 5.07 & $\mathrm{C}_{53} \mathrm{H}_{88} \mathrm{O}_{23}$ & 1091.56327 & 1091.52698 & & $1023,1007,965,783$ & Yesanchinoside G & PJ \\
\hline 2 & 5.31 & $\mathrm{C}_{48} \mathrm{H}_{82} \mathrm{O}_{19}$ & 961.53666 & 961.53546 & 1007.54340 & $799,781,637,619$ & G-Re1/G-Re2/G-Re3/NG-N/NG-M isomer & PJ, PZ \\
\hline 3 & 5.36 & $\mathrm{C}_{53} \mathrm{H}_{90} \mathrm{O}_{22}$ & 1077.58400 & 1077.54590 & & $945,783,637,475,391,191$ & Floral G-M/Floral G-N & PJ, PM, PZ \\
\hline 4 & 5.44 & $\mathrm{C}_{53} \mathrm{H}_{88} \mathrm{O}_{23}$ & 1091.56327 & 1091.52454 & & $929,767,473$ & Yesanchinoside G isomer & PJ, PM, PZ \\
\hline 5 & 5.61 & $\mathrm{C}_{47} \mathrm{H}_{80} \mathrm{O}_{19}$ & 947.52101 & 947.52203 & 993.52789 & $815,653,579,491,391$ & Vina G-R6/Yesanchinoside C & PZ \\
\hline 6 & 5.64 & $\mathrm{C}_{48} \mathrm{H}_{82} \mathrm{O}_{19}$ & 961.53666 & 961.53775 & 1007.54224 & $799,781,637,475,391$ & 20-glc-G-Rf & PJ, PM \\
\hline 7 & 5.74 & $\mathrm{C}_{48} \mathrm{H}_{82} \mathrm{O}_{19}$ & 961.53666 & 961.53503 & 1007.54285 & $943,931,799,637$ & G-Re1/G-Re2/G-Re3/NG-N/NG-M isomer & PJ, PM, PZ \\
\hline 8 & 5.75 & $\mathrm{C}_{54} \mathrm{H}_{92} \mathrm{O}_{23}$ & 1107.59457 & 1107.59314 & 1153.60094 & $945,783,637,475,391$ & Yesanchinoside E & PM, PZ \\
\hline 9 & 5.92 & $\mathrm{C}_{41} \mathrm{H}_{70} \mathrm{O}_{14}$ & 785.46818 & 785.46246 & 831.47443 & $739,653,491,391$ & Majonoside R2 isomer & PJ \\
\hline 10 & 5.93 & $\mathrm{C}_{48} \mathrm{H}_{82} \mathrm{O}_{19}$ & 961.53666 & 961.53602 & 1007.54651 & $799,781,637,475,391$ & 20-glc-G-Rf isomer & PJ \\
\hline 11 & 5.94 & $\mathrm{C}_{53} \mathrm{H}_{90} \mathrm{O}_{22}$ & 1077.58400 & 1077.58337 & 1123.58960 & $945,783,637,475,391,191$ & FloralG-M/Floral G-N & PM \\
\hline 12 & 5.99 & $\mathrm{C}_{41} \mathrm{H}_{70} \mathrm{O}_{14}$ & 785.46818 & 785.46429 & 831.47418 & $739,653,491,391$ & Majonoside R2 isomer & PJ, PZ \\
\hline $13^{*}$ & 6.01 & $\mathrm{C}_{47} \mathrm{H}_{80} \mathrm{O}_{18}$ & 931.52609 & 931.52631 & 977.53192 & $799,769,637,475$ & NG-R1 & PJ, PM, PZ \\
\hline 14 & 6.09 & $\mathrm{C}_{42} \mathrm{H}_{72} \mathrm{O}_{14}$ & 799.48383 & 799.48421 & & $667,653,491,455,391$ & Pseudo G-F11 isomer & PJ, PM \\
\hline 15 & 6.10 & $\mathrm{C}_{51} \mathrm{H}_{82} \mathrm{O}_{18}$ & 981.54174 & 981.54567 & & $793,763,619,581,455,371$ & Pseudo G-RT1 butyl ester & $\mathrm{PM}$ \\
\hline 16 & 6.11 & $\mathrm{C}_{56} \mathrm{H}_{94} \mathrm{O}_{24}$ & 1149.60513 & 1149.60181 & 1195.61084 & $1149,1107,961,783,637,475,391$ & Acetyl Yesanchinoside E & PM \\
\hline $17^{*}$ & 6.13 & $\mathrm{C}_{48} \mathrm{H}_{82} \mathrm{O}_{18}$ & 945.54174 & 945.53772 & 991.54773 & $783,637,619,475,391,205$ & G-Re & PJ, PM, PZ \\
\hline $18^{*}$ & 6.18 & $\mathrm{C}_{42} \mathrm{H}_{72} \mathrm{O}_{14}$ & 799.48383 & 799.48541 & 845.48975 & $637,475,391$ & G-Rg1 & PJ, PM, PZ \\
\hline 19 & 6.27 & $\mathrm{C}_{45} \mathrm{H}_{74} \mathrm{O}_{17}$ & 885.48423 & 885.48517 & & $845,829,781,637,619,475,391$ & Malonyl-G-Rg1 & PJ, PZ \\
\hline 20 & 6.29 & $\mathrm{C}_{58} \mathrm{H}_{98} \mathrm{O}_{26}$ & 1209.62626 & 1209.59033 & & $1165,781,619,459$ & NG-Fc & PM \\
\hline 21 & 6.30 & $\mathrm{C}_{47} \mathrm{H}_{80} \mathrm{O}_{19}$ & 947.52101 & 947.52191 & & $815,653,579,491,391$ & Vina G-R6/Yesanchinoside C & PJ, PM, PZ \\
\hline 22 & 6.32 & $\mathrm{C}_{51} \mathrm{H}_{84} \mathrm{O}_{21}$ & 1031.54214 & 1031.54419 & & $987,945,637,475,391$ & Malonyl G-Re & PJ, PZ \\
\hline 23 & 6.34 & $\mathrm{C}_{41} \mathrm{H}_{70} \mathrm{O}_{14}$ & 785.46818 & 785.46503 & 831.47430 & $739,653,491,391$ & Majonoside R2 isomer & PJ, PM, PZ \\
\hline $24 *$ & 6.40 & $\mathrm{C}_{42} \mathrm{H}_{72} \mathrm{O}_{14}$ & 799.48383 & 799.48566 & 799.47870 & $754,653,491,473,455$ & Pseudo G-F11 & PJ, PM \\
\hline 25 & 6.46 & $\mathrm{C}_{48} \mathrm{H}_{82} \mathrm{O}_{19}$ & 961.53666 & 961.53662 & 1007.54055 & $799,781,637,499$ & G-Re1/G-Re2/G-Re3/NG-N/NG-M isomer & PJ, PM, PZ \\
\hline 26 & 6.53 & $\mathrm{C}_{50} \mathrm{H}_{84} \mathrm{O}_{19}$ & 987.55231 & 987.55084 & 1033.55835 & $945,791,763,637,475,391,275$ & Acetyl G-Re & PM, PJ, PZ \\
\hline 27 & 6.54 & $\mathrm{C}_{44} \mathrm{H}_{74} \mathrm{O}_{15}$ & 841.49440 & 841.49204 & 887.50067 & $841,795,637,475,391$ & Acetyl-Rg1 & PJ, PZ \\
\hline 28 & 6.55 & $\mathrm{C}_{42} \mathrm{H}_{72} \mathrm{O}_{15}$ & 815.47875 & 815.47961 & 861.48315 & 637 & Floralquinquenoside B & PJ, PM, PZ \\
\hline 29 & 6.60 & $\mathrm{C}_{48} \mathrm{H}_{82} \mathrm{O}_{19}$ & 961.53666 & 961.53543 & 1007.54660 & $943,799,781,457$ & G-Re1/G-Re2/G-Re3/NG-N/NG-M isomer & $\mathrm{PJ}$ \\
\hline 30 & 6.67 & $\mathrm{C}_{59} \mathrm{H}_{100} \mathrm{O}_{27}$ & 1239.63682 & 1239.63452 & 1285.64172 & $1107,1059,945,783,621,459$ & NG-R4/NG-Fa & PJ, PM, PZ \\
\hline 31 & 6.68 & $\mathrm{C}_{56} \mathrm{H}_{92} \mathrm{O}_{25}$ & 1163.58439 & 1163.54858 & 1209.59326 & $1117,955,793,621,537,459,351$ & Malonyl G-Rc & PM \\
\hline 32 & 6.69 & $\mathrm{C}_{48} \mathrm{H}_{80} \mathrm{O}_{19}$ & 959.52101 & 959.52148 & 1005.54272 & $797,779,635,617,473,455$ & NG-G & PM, PZ \\
\hline 33 & 6.76 & $\mathrm{C}_{42} \mathrm{H}_{72} \mathrm{O}_{15}$ & 815.47875 & 815.47610 & 861.48440 & $653,491,415$ & Floralquinquenoside D & PJ, PM \\
\hline 34 & 6.79 & $\mathrm{C}_{43} \mathrm{H}_{72} \mathrm{O}_{15}$ & 827.47875 & 827.47998 & & $695,491,455$ & Vina G-R2 & PZ \\
\hline 35 & 6.82 & $\mathrm{C}_{58} \mathrm{H}_{98} \mathrm{O}_{26}$ & 1209.62626 & 1209.62500 & 1255.63147 & $1077,1047,945,783,621,459$ & G-Ra2 & PJ, PM, PZ \\
\hline 36 & 6.84 & $\mathrm{C}_{63} \mathrm{H}_{106} \mathrm{O}_{30}$ & 1341.66852 & 1341.66748 & 1387.67493 & $1209,1077,945,783,621,459$ & NG-Q & $\mathrm{PJ}$ \\
\hline 37 & 6.86 & $\mathrm{C}_{44} \mathrm{H}_{74} \mathrm{O}_{15}$ & 841.49440 & 841.49880 & 887.50098 & $799,637,475,391$ & Acetyl-Rf & PJ, PM, PZ \\
\hline 38 & 6.89 & $\mathrm{C}_{59} \mathrm{H}_{100} \mathrm{O}_{27}$ & 1239.63682 & 1239.63538 & 1285.64185 & $1077,1059,945,783,765,459$ & NG-R4/NG-Fa & PJ, PM, PZ \\
\hline $39 *$ & 6.92 & $\mathrm{C}_{54} \mathrm{H}_{92} \mathrm{O}_{23}$ & 1107.59457 & 1107.59302 & 1153.59973 & $945,783,621,459$ & G-Rb1 & PJ, PM, PZ \\
\hline
\end{tabular}


Table 3. Cont

\begin{tabular}{|c|c|c|c|c|c|c|c|c|}
\hline \multirow[t]{2}{*}{ No. } & \multirow[t]{2}{*}{$T_{R} / \min$} & \multirow{2}{*}{$\begin{array}{c}\text { Formula } \\
\text { Calculated }\end{array}$} & \multicolumn{2}{|c|}{$[\mathbf{M}-\mathbf{H}]^{-}$} & \multirow[t]{2}{*}{$\mathrm{M}+\mathrm{HCOO}^{-}$} & \multirow[t]{2}{*}{ MS/MS } & \multirow[t]{2}{*}{ Identification } & \multirow[t]{2}{*}{ Sample } \\
\hline & & & Measured & & & & & \\
\hline 40 & 6.95 & $\mathrm{C}_{57} \mathrm{H}_{94} \mathrm{O}_{26}$ & 1193.59496 & 1193.59509 & & $1149,1107,945,783,621,459,375$ & Malonyl G-Rb1 & PJ, PM, PZ \\
\hline 41 * & 6.96 & $\mathrm{C}_{42} \mathrm{H}_{72} \mathrm{O}_{14}$ & 799.48383 & 799.48175 & 845.48956 & $637,475,391$ & G-Rf & PJ, PM, PZ \\
\hline 42 & 6.97 & $\mathrm{C}_{58} \mathrm{H}_{98} \mathrm{O}_{26}$ & 1209.62626 & 1209.62488 & 1255.63074 & $1077,1047,915,945,783,621,459$ & G-Ra1 & PJ, PZ \\
\hline 43 & 6.99 & $\mathrm{C}_{41} \mathrm{H}_{70} \mathrm{O}_{14}$ & 785.46818 & 785.46852 & 831.47382 & 653,491 & Majonoside R2 & PJ, PM, PZ \\
\hline 44 & 7.00 & $\mathrm{C}_{53} \mathrm{H}_{84} \mathrm{O}_{23}$ & 1087.53196 & 1087.53149 & & $955,925,793,569,497,455,283$ & Stipuleanoside R2 & PJ, PM, PZ \\
\hline 45 & 7.03 & $\mathrm{C}_{61} \mathrm{H}_{100} \mathrm{O}_{29}$ & 1295.62665 & 1295.62537 & & $1251,1209,1077,945,783,621,459$ & Malonyl G-Ra2 & PJ, PZ \\
\hline 46 & 7.04 & $\mathrm{C}_{42} \mathrm{H}_{72} \mathrm{O}_{14}$ & 799.48383 & 799.48041 & 845.48962 & $637,619,499,457$ & Majoroside F2/F3/F4 & PJ, PM \\
\hline 47 & 7.04 & $\mathrm{C}_{48} \mathrm{H}_{76} \mathrm{O}_{19}$ & 955.48971 & 955.49017 & 1001.49530 & $793,631,455,349$ & G-Ro isomer & PJ, PM, PZ \\
\hline $48 *$ & 7.05 & $\mathrm{C}_{53} \mathrm{H}_{90} \mathrm{O}_{22}$ & 1077.58400 & 1077.58130 & 1123.58936 & $945,783,621,459$ & G-Rc & PM, PZ \\
\hline 49 & 7.06 & $\mathrm{C}_{53} \mathrm{H}_{84} \mathrm{O}_{23}$ & 1087.53196 & 1087.53235 & 1133.53625 & $925,731,569,459$ & Stipuleanoside $\mathrm{R} 2$ isomer & PZ \\
\hline 50 & 7.07 & $\mathrm{C}_{56} \mathrm{H}_{92} \mathrm{O}_{25}$ & 1163.58439 & 1163.58459 & & $1119,1077,945,783,621,459$ & Malonyl G-Rb2 & PM \\
\hline 51 & 7.08 & $\mathrm{C}_{57} \mathrm{H}_{94} \mathrm{O}_{26}$ & 1193.59496 & 1193.59497 & & $1149,1107,945,783,621,459,375$ & Malonyl G-Rb1 isomer & PJ, PM \\
\hline $52 *$ & 7.09 & $\mathrm{C}_{41} \mathrm{H}_{70} \mathrm{O}_{13}$ & 769.47327 & 769.47125 & 815.47961 & $637,485,475,325,311$ & G-F3 & PJ, PM, PZ \\
\hline 53 & 7.09 & $\mathrm{C}_{56} \mathrm{H}_{94} \mathrm{O}_{24}$ & 1149.60513 & 1149.60083 & 1195.60669 & $1107,945,783,621,475$ & Yesanchinoside F & PJ, PM \\
\hline 54 * & 7.15 & $\mathrm{C}_{53} \mathrm{H}_{90} \mathrm{O}_{22}$ & 1077.58400 & 1077.58179 & 1123.58923 & $945,915,783,621,459$ & $\mathrm{G}-\mathrm{Rb} 2$ & PJ, PZ \\
\hline $55^{*}$ & 7.16 & $\mathrm{C}_{48} \mathrm{H}_{76} \mathrm{O}_{19}$ & 955.48971 & 955.48987 & 1001.49347 & $793,731,659,631,455$ & G-Ro & PJ, PM, PZ \\
\hline 56 & 7.18 & $\mathrm{C}_{61} \mathrm{H}_{100} \mathrm{O}_{29}$ & 1295.62665 & 1295.62561 & & $1107,945,783,621,459$ & Malonyl G-Ra1 & $\mathrm{PJ}$ \\
\hline 57 & 7.19 & $\mathrm{C}_{56} \mathrm{H}_{92} \mathrm{O}_{25}$ & 1163.58439 & 1163.58435 & & $1119,1077,945,783,621,459,293$ & Malonyl G-Rb3 & PJ, PM \\
\hline 58 * & 7.21 & $\mathrm{C}_{42} \mathrm{H}_{72} \mathrm{O}_{13}$ & 783.48892 & 783.48627 & 829.49475 & $637,619,475,457,391,205,161$ & G-Rg2 & PJ, PM, PZ \\
\hline 59 & 7.22 & $\mathrm{C}_{45} \mathrm{H}_{74} \mathrm{O}_{17}$ & 885.48423 & 885.48474 & & $829,799,637,475$ & Malonyl-G-Rf & PM \\
\hline $60 *$ & 7.23 & $\mathrm{C}_{53} \mathrm{H}_{90} \mathrm{O}_{22}$ & 1077.58400 & 1077.58289 & 1123.58960 & $945,915,783,621,459$ & G-Rb3 & PZ \\
\hline $61 *$ & 7.32 & $\mathrm{C}_{47} \mathrm{H}_{74} \mathrm{O}_{18}$ & 925.47914 & 925.47894 & 971.48279 & $873,793,612,569,455$ & CS-IV & PJ, PZ \\
\hline $62 *$ & 7.35 & $\mathrm{C}_{36} \mathrm{H}_{62} \mathrm{O}_{9}$ & 637.43101 & 637.42828 & 683.43695 & $457,391,283,255$ & G-Rh1 & PJ, PM, PZ \\
\hline 63 & 7.37 & $\mathrm{C}_{48} \mathrm{H}_{82} \mathrm{O}_{19}$ & 961.53666 & 961.53674 & 1007.54553 & $815,781,499$ & G-Re1/G-Re2/G-Re3/NG-N/NG-M isomer & PM \\
\hline $64^{*}$ & 7.43 & $\mathrm{C}_{48} \mathrm{H}_{82} \mathrm{O}_{18}$ & 945.54174 & 945.53973 & 991.54706 & $783,621,459$ & G-Rd & PJ, PM, PZ \\
\hline 65 & 7.44 & $\mathrm{C}_{51} \mathrm{H}_{84} \mathrm{O}_{21}$ & 1031.54214 & 1031.54248 & & $987,945,783,621,459,375$ & Malonyl G-Rd & PJ, PM, PZ \\
\hline 66 & 7.46 & $\mathrm{C}_{50} \mathrm{H}_{84} \mathrm{O}_{19}$ & 987.55231 & 987.55151 & 1033.55066 & $945,783,621,459$ & Acetyl G-Rd & PM, PJ \\
\hline $67 *$ & 7.53 & $\mathrm{C}_{42} \mathrm{H}_{66} \mathrm{O}_{14}$ & 793.43688 & 793.43701 & 839.44269 & $631,569,509,497,455$ & CS-IVa & PJ, PM, PZ \\
\hline 68 & 7.60 & $\mathrm{C}_{51} \mathrm{H}_{84} \mathrm{O}_{21}$ & 1031.54214 & 1031.54224 & & $987,945,783,621,459,375$ & Malonyl G-Rd isomer & PM, PJ \\
\hline 69 * & 7.61 & $\mathrm{C}_{36} \mathrm{H}_{62} \mathrm{O}_{9}$ & 637.43101 & 637.43042 & 683.43707 & 475,391 & G-F1 & PJ, PM, PZ \\
\hline 70 & 7.63 & $\mathrm{C}_{48} \mathrm{H}_{82} \mathrm{O}_{18}$ & 945.54174 & 945.53990 & 991.54767 & $899,783,855,793,621,459$ & Gypenoside XVII & PJ, PM, PZ \\
\hline 71 & 7.64 & $\mathrm{C}_{53} \mathrm{H}_{90} \mathrm{O}_{23}$ & 1093.57892 & 1093.57825 & & $1047,915,783,621,459$ & Gypenoside LVI/LXVII/Floral G-P & PJ, PZ \\
\hline 72 & 7.66 & $\mathrm{C}_{49} \mathrm{H}_{78} \mathrm{O}_{19}$ & 969.50536 & 969.99005 & 1015.51208 & $807,631,537,455,393$ & G-Ro methyl ester & PM, PZ \\
\hline 73 & 7.73 & $\mathrm{C}_{47} \mathrm{H}_{80} \mathrm{O}_{17}$ & 915.53118 & 915.53667 & 961.53442 & $783,621,459,375$ & NG-Fe & PJ, PM, PZ \\
\hline 74 & 7.79 & $\mathrm{C}_{38} \mathrm{H}_{64} \mathrm{O}_{10}$ & 679.44157 & 679.44607 & & $633,611,475,391$ & Acety G-Rh1 & PJ, PM \\
\hline 75 & 7.82 & $\mathrm{C}_{47} \mathrm{H}_{80} \mathrm{O}_{17}$ & 915.53118 & 915.52783 & 961.53748 & $783,621,459,375$ & CS-III & PJ, PZ \\
\hline 76 & 7.82 & $\mathrm{C}_{48} \mathrm{H}_{82} \mathrm{O}_{19}$ & 961.53666 & 961.53802 & 1007.53357 & $931,799,619$ & G-Re1/G-Re2/G-Re3/NG-N/NG-M isomer & PJ, PM, PZ \\
\hline 77 & 7.90 & $\mathrm{C}_{38} \mathrm{H}_{64} \mathrm{O}_{10}$ & 679.44157 & 679.44049 & & $633,475,391$ & Acety G-F1 & PJ, PM \\
\hline 78 & 7.92 & $\mathrm{C}_{41} \mathrm{H}_{70} \mathrm{O}_{13}$ & 769.47327 & 769.43805 & & $679,637,475,391$ & Pseudo G-RT3/G-F5 & PM, PZ \\
\hline 79 & 7.94 & $\mathrm{C}_{48} \mathrm{H}_{82} \mathrm{O}_{19}$ & 961.53666 & 961.53735 & 1007.54629 & $915,621,499$ & G-Re1/G-Re2/G-Re3/NG-N/NG-M isomer & PM, PJ, PZ \\
\hline 80 & 8.11 & $\mathrm{C}_{43} \mathrm{H}_{68} \mathrm{O}_{14}$ & 807.45253 & 807.41650 & 853.46051 & $793,631,455$ & CS-IVa methyl ester & PJ, PM, PZ \\
\hline
\end{tabular}


Table 3. Cont

\begin{tabular}{|c|c|c|c|c|c|c|c|c|}
\hline \multirow[t]{2}{*}{ No. } & \multirow[t]{2}{*}{$\mathrm{T}_{\mathrm{R}} / \mathrm{min}$} & \multirow{2}{*}{$\begin{array}{c}\text { Formula } \\
\text { Calculated }\end{array}$} & \multicolumn{2}{|c|}{$[\mathbf{M}-\mathbf{H}]^{-}$} & \multirow{3}{*}{$\frac{\left[\mathrm{M}+\mathrm{HCOO}^{-}\right.}{839.44141}$} & \multirow[t]{2}{*}{ MS/MS } & \multirow[t]{2}{*}{ Identification } & \multirow[t]{2}{*}{ Sample } \\
\hline & & & $\begin{array}{l}\text { Measured } \\
79343688\end{array}$ & & & & & \\
\hline 81 & 8.17 & $\mathrm{C}_{42} \mathrm{H}_{66} \mathrm{O}_{14}$ & 793.43688 & 793.43787 & & $631,613,569,455$ & CS-II & PM, PZ \\
\hline 82 & 8.19 & $\mathrm{C}_{47} \mathrm{H}_{74} \mathrm{O}_{18}$ & 925.47914 & 925.47986 & & $793,731,569,497,455$ & CS-Ib & PJ, PM, PZ \\
\hline 83 & 8.31 & $\mathrm{C}_{47} \mathrm{H}_{74} \mathrm{O}_{18}$ & 925.47914 & 925.47998 & 971.48346 & $793,763,631,455$ & pseudo G-RT1/Stipuleanoside R1 & PJ, PM, PZ \\
\hline 84 & 8.38 & $\mathrm{C}_{42} \mathrm{H}_{66} \mathrm{O}_{14}$ & 793.43688 & 793.43707 & 839.44318 & $731,631,613,569,455$ & Zingibroside R1 & PJ, PM, PZ \\
\hline $85 *$ & 8.42 & $\mathrm{C}_{42} \mathrm{H}_{72} \mathrm{O}_{13}$ & 783.48892 & 783.48669 & 829.49506 & 621,459 & (20S) G-Rg3 & PM \\
\hline 86 & 8.67 & $\mathrm{C}_{41} \mathrm{H}_{64} \mathrm{O}_{13}$ & 763.42632 & 763.42659 & 809.43291 & $631,613,569,455,325$ & Pseudo G-RP1 & PJ, PM \\
\hline 87 & 8.68 & $\mathrm{C}_{42} \mathrm{H}_{70} \mathrm{O}_{12}$ & 765.47835 & 765.43286 & & $603,593,441$ & G-Rk1 & PJ, PM, PZ \\
\hline $88^{*}$ & 8.71 & $\mathrm{C}_{42} \mathrm{H}_{72} \mathrm{O}_{13}$ & 783.48892 & 783.48826 & 829.49457 & 621,459 & G-F2 & PJ, PM, PZ \\
\hline 89 & 8.78 & $\mathrm{C}_{42} \mathrm{H}_{72} \mathrm{O}_{13}$ & 783.48892 & 783.48615 & 829.49524 & $752,599,459$ & (20R)-G-Rg3 & PJ, PM, PZ \\
\hline 90 & 8.89 & $\mathrm{C}_{41} \mathrm{H}_{64} \mathrm{O}_{13}$ & 763.42632 & 763.42631 & & $613,569,497,455,405$ & Pseudo G-RP1 isomer & PJ, PM, PZ \\
\hline 91 & 8.90 & $\mathrm{C}_{42} \mathrm{H}_{70} \mathrm{O}_{12}$ & 765.47835 & 765.43274 & & $719,673,603,573,459$ & G-Rg5 & PJ, PM, PZ \\
\hline 92 & 8.95 & $\mathrm{C}_{42} \mathrm{H}_{70} \mathrm{O}_{11}$ & 749.48344 & 749.48045 & & 617,455 & Pjs-4 & PJ \\
\hline 93 & 9.11 & $\mathrm{C}_{58} \mathrm{H}_{96} \mathrm{O}_{24}$ & 1175.62078 & 1175.67310 & 1221.67749 & $1159,1095,955,793,613,569,459$ & G-Ra6 & PJ \\
\hline 94 & 9.64 & $\mathrm{C}_{37} \mathrm{H}_{62} \mathrm{O}_{7}$ & 617.44118 & 617.44056 & 663.00000 & 455,359 & Oleanolic acid 28-O- $\beta$-D-glucopyranoside & PJ, PM, PZ \\
\hline 95 & 9.71 & $\mathrm{C}_{44} \mathrm{H}_{74} \mathrm{O}_{15}$ & 841.49440 & 841.49487 & & $795,633,491,471$ & Vina G-R1 & PJ, PM, PZ \\
\hline $96^{*}$ & 9.84 & $\mathrm{C}_{30} \mathrm{H}_{52} \mathrm{O}_{4}$ & 475.37819 & 475.36335 & & 459,391 & PPT & PJ, PM, PZ \\
\hline 97 & 9.87 & $\mathrm{C}_{36} \mathrm{H}_{60} \mathrm{O}_{7}$ & 603.42553 & 603.33807 & 649.34393 & 441,279 & G-Rk2 & PJ, PM \\
\hline 98 & 10.08 & $\mathrm{C}_{36} \mathrm{H}_{62} \mathrm{O}_{8}$ & 621.43610 & 621.43738 & 667.44208 & $459,375,325,311$ & $\mathrm{G}-\mathrm{K}$ & PJ, PM \\
\hline 99 * & 10.18 & $\mathrm{C}_{36} \mathrm{H}_{62} \mathrm{O}_{8}$ & 621.43610 & 621.43530 & 667.44232 & $459,375,283,255$ & G-Rh2 & PJ, PM, PZ \\
\hline 100 & 10.34 & $\mathrm{C}_{36} \mathrm{H}_{62} \mathrm{O}_{10}$ & 653.42592 & 653.42682 & & 491 & Pseudo G-RT4 & PJ, PM \\
\hline $101 *$ & 10.94 & $\mathrm{C}_{30} \mathrm{H}_{52} \mathrm{O}_{3}$ & 459.38327 & 459.39596 & & 375,329 & PPD & PJ, PM \\
\hline
\end{tabular}

* identified with a standard reference. 

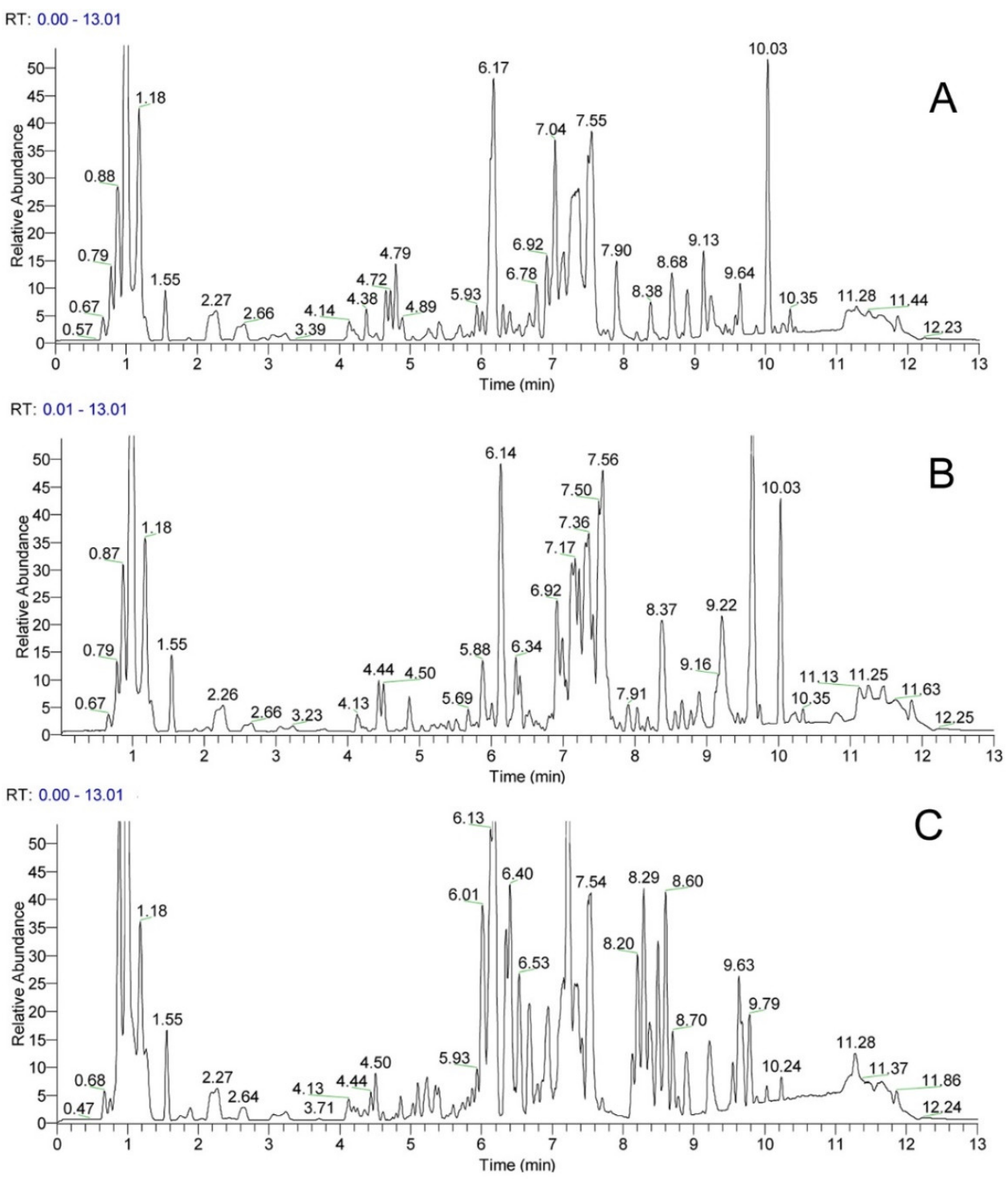

Figure 2. The base peak chromatogram of the roots and rhizomes of PJ (A), PM (B), and PZ (C) in negative mode. Panax japonicas (PJ), Panax japonicus var. major (PM), Panax zingiberensis (PZ).

Phytochemistry research studies have demonstrated that the chemical constituents in the Panax genus are very complex [1,22,23]. Ginsenosides are the major effective components. Currently, hundreds of ginsenosides have been isolated and unambiguously characterized from these Panax species, especially from P. ginseng, P. quinquefolium, and P. notoginseng. Furthermore, the fragmentation pathways of numerous ginsenosides have been reported by numerous research studies [24,25], which makes it easier to detect and identify secondary metabolites from Panax species using the UHPLC-Q-Exactive/HRMS technique. In the present study, ESI-MS on negative ion mode was used for compound detection and characterization due to its high sensitivity and sensitivity, as well as clear mass spectra in the negative ion mode. A total of 101 compounds were detected and tentatively identified, including 82 from PJ, 78 from PM, and 67 from PZ. Among them, 22 ginsenosides, including ginsenosides G-Rg1, G-Re, G-Rb1, G-Rc, G-Rb2, G-Rb3, G-Rf, G-Rd, G-Rg2, G-Rg3, G-F3, G-Rh1, G-Rh2, G-Ro, G-F1, G-F2, NG-R1, pseudo G-F11, PPD, PPT, CS-IV, and CS-IVa, were unambiguously identified by comparing their retention times and fragment ions with the reference standards. The others were tentatively assigned by the empirical molecular formula, theoretical molecular mass, and MS/MS fragment ions as well as the retention sequence of isomeric ginsenosides. 
In the MS spectra, most of the ginsenosides showed deprotonated ions $[\mathrm{M}-\mathrm{H}]^{-}$and/or formic acid adduct ions $[\mathrm{M}+\mathrm{HCOO}]^{-}$in the negative ion mode. However, it is worth noting that the malonyl-ginsenosides could not produce adduct ions $[\mathrm{M}+\mathrm{HCOO}]^{-}$because malonyl-ginsenosides are unstable. It is easy to lose $\mathrm{CO}_{2}$ from the deprotonated molecules of these compounds under demalonylation, which results in the detection of the peaks in the quasi-molecular ions of [M- $\mathrm{H}-$ $\left.\mathrm{CO}^{-}\right]^{-}$and $\left[\mathrm{M}-\mathrm{H}-\mathrm{Malonyl}^{-}\right.$, which were found in compounds $19,22,31,40,45,46,50,51,56$, 57, 59, 65, and 68. Additionally, according to the negative MS, MS/MS spectra of the ginsenosides, the deprotonated ions and their product ions exhibited the common fragmentation pattern that corresponds to the successive or simultaneous loss of glycosidic units, such as the glucosyl (Glc) group (162 Da), the rhamnosyl (Rha) group (146 Da), the xylose (Xyl)/arabinose(Ara) group (132 Da), and the glucuronyl (Glu A) group (176 Da), at the C-20, C-3, or C-6 sites until the formation of an aglycone ion. The characteristic ions at $m / z 475\left(\mathrm{C}_{30} \mathrm{H}_{51} \mathrm{O}_{4}\right)$ and $m / z 459\left(\mathrm{C}_{30} \mathrm{H}_{51} \mathrm{O}_{3}\right)$ were observed in the PPTs (such as compounds $3,6,8,10,11,13,17,18,41,53,62,69$, and 78 ) and PPDs (such as compounds 20, 30, 31, $35,36,39,42,48,54,60,64,70,71,75,85,88,89,91,93$, and 98$)$, respectively. As shown in Figure 3A,B, G-Rb1 produced [(20S)-protopanaxadiol $-\mathrm{H}]^{-}$at $m / z 459.38260\left(\mathrm{C}_{30} \mathrm{H}_{51} \mathrm{O}_{3}\right)$ in the MS/MS spectrum by the successive loss of four Glc (162 Da), and NG-R1 gave the [(20S)-protopanaxatriol $-\mathrm{H}]^{-}$at $m / z 475.37848\left(\mathrm{C}_{30} \mathrm{H}_{51} \mathrm{O}_{4}\right)$ via the successive elimination of one $\mathrm{Xyl}$ and two Glc. Moreover, the OAs displayed an aglycone ion at $m / z 455\left(\mathrm{C}_{30} \mathrm{H}_{47} \mathrm{O}_{3}\right)$ corresponding to [oleanolic acid $\left.-\mathrm{H}\right]^{-}$, which was visible for compounds $47,55,61,67,80,81,82,84$, and 94 . Figure 3C,D show the MS/MS spectra of G-Ro (55) and CS-IV (61). These two saponins produced [oleanolic acid - H] ${ }^{-}$at $m / z 455\left(\mathrm{C}_{30} \mathrm{H}_{47} \mathrm{O}_{3}\right)$ after the successive loss of Glc, and/or Ara, and Glu A.
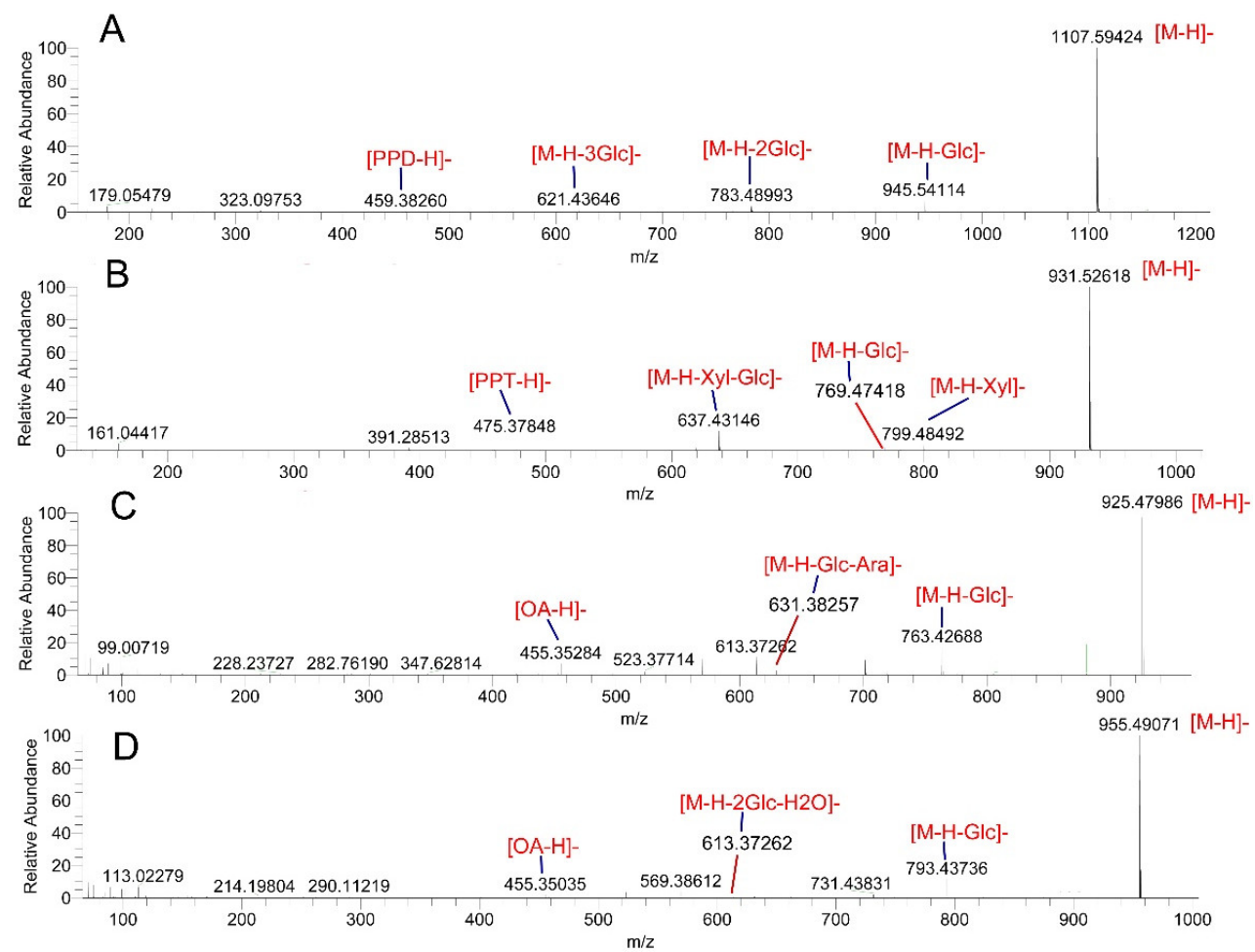

Figure 3. The MS/MS spectrum of ginsenoside -Rb1 (A), ginsenoside -R1 (B), ginsenoside -Ro (C), and chikusetsu saponin -IV (D).

Notably, some Octillol-type triterpenoid saponins were also detected and identified in the three ginseng herbs. For example, compounds 24 (Pseudo G-F11), 34 (Vina G-R1), 95 (Vina G-R2), 100 (Pseudo G-RT4), 5, and 21 (Vina G-R6/Yesanchinoside C) were observed at $m / z 491\left(\mathrm{C}_{30} \mathrm{H}_{51} \mathrm{O}_{5}\right)$ via the loss of a different sugar moiety, corresponding to the deprotonated ions of Octillol-type aglycone. 


\subsection{Validation of the Quantitative Analytical Method}

The HPLC-ESI-MS/MS quantitative analysis method was validated. The regression equations, coefficient of determination, linear ranges, LODs, and LOQs for the quantitative analysis of the 16 reference ginsenosides are shown in Table 4 . The calibration curves for all 16 reference substances showed good linear regression $(r>0.999)$ within the test ranges. The LODs of the 16 reference compounds were estimated to be $0.13-2.22 \mathrm{ng} / \mathrm{mL}$, whereas the LOQs were $0.31-5.90 \mathrm{ng} / \mathrm{mL}$. The precision, repeatability, stability, and recovery are listed in Table 5 . The precision of the quantitative method was determined; the validation studies showed that the relative standard deviation (RSD) was less than $4.87 \%$, and the repeatability of the method was very good (RSD $<4.93 \%$ ). The RSD of the storage stability was less than $4.60 \%$ in $24 \mathrm{~h}$. The recovery was in the range of $99.25-104.10 \%$ with an RSD of less than $3.45 \%$. The established HPLC-ESI-MS/MS method was accurate and reliable and is therefore appropriate for quantitative analysis.

Table 4. The regression equations, linear range, limit of detection (LOD), and limit of quantification (LOQ) of the 16 analytes.

\begin{tabular}{|c|c|c|c|c|c|c|}
\hline No. & Analyte & $\begin{array}{l}\text { Regression } \\
\text { Equations }\end{array}$ & $\begin{array}{c}\text { Correlation } \\
\text { Coefficients (r) }\end{array}$ & $\begin{array}{l}\text { Linear Range } \\
\left(\mathrm{ng} \times \mathrm{mL}^{-1}\right)\end{array}$ & $\begin{array}{c}\text { LOD } \\
\left(\mathrm{ng} \times \mathrm{mL}^{-1}\right)\end{array}$ & $\begin{array}{c}\mathrm{LOQ} \\
\left(\mathrm{ng} \times \mathrm{mL}^{-1}\right)\end{array}$ \\
\hline 1 & N-R1 & $Y=22.1 X+29.4$ & 0.9991 & $1.56 \sim 1560$ & 0.61 & 1.22 \\
\hline 2 & G-Rg1 & $\mathrm{Y}=7.7 \mathrm{X}+193$ & 0.9999 & $3.91 \sim 3910$ & 1.91 & 3.81 \\
\hline 3 & G-Re & $Y=18 X+103$ & 0.9998 & $6.25 \sim 6250$ & 0.38 & 1.22 \\
\hline 4 & G-Rf & $Y=53.4 X+20$ & 0.9998 & $0.78 \sim 780$ & 0.19 & 0.38 \\
\hline 5 & G-F3 & $Y=12.3 X+8.91$ & 0.9997 & $1.17 \sim 1170$ & 0.36 & 0.91 \\
\hline 6 & G-Rg2 & $Y=71.7 X+50.6$ & 0.9994 & $7.03 \sim 7030$ & 0.21 & 0.86 \\
\hline 7 & G-Rh1 & $Y=2.92 X-1.56$ & 0.9992 & $6.24 \sim 1560$ & 2.22 & 5.90 \\
\hline 8 & G-Rb1 & $Y=7.5 X-105$ & 0.9996 & $3.15 \sim 3130$ & 1.53 & 3.05 \\
\hline 9 & G-Ro & $Y=27.8 X+3750$ & 0.9992 & $9.38 \sim 9380$ & 0.29 & 0.88 \\
\hline 10 & G-Rc & $Y=7.97 X+65.1$ & 0.9998 & $1.56 \sim 1560$ & 0.61 & 1.22 \\
\hline 11 & G-Rb2 & $Y=12.3 X+78.7$ & 0.9998 & $1.56 \sim 1560$ & 0.41 & 1.22 \\
\hline 12 & G-Rb3 & $Y=15.1 X+56.6$ & 0.9996 & $1.56 \sim 1560$ & 0.41 & 1.38 \\
\hline 13 & CS-IV & $Y=79.3 X+2610$ & 0.9997 & $6.25 \sim 6250$ & 0.16 & 0.31 \\
\hline 14 & CS-IVa & $\mathrm{Y}=13.4 \mathrm{X}+844$ & 0.9991 & $7.81 \sim 7810$ & 0.13 & 0.38 \\
\hline 15 & G-Rd & $\mathrm{Y}=14 \mathrm{X}+188$ & 0.9993 & $1.56 \sim 1560$ & 0.41 & 1.07 \\
\hline 16 & G-Rg3 & $Y=24.1 X-17.1$ & 0.9995 & $0.78 \sim 780$ & 0.25 & 0.76 \\
\hline
\end{tabular}

Table 5. The precision, repeatability, stability, and recovery of the 16 analytes.

\begin{tabular}{ccccccc}
\hline No. & Analyte & $\begin{array}{c}\text { Precision } \\
(\text { RSD, } \mathbf{\%}, \mathbf{n}=\mathbf{6})\end{array}$ & $\begin{array}{c}\text { Repeatability } \\
(\mathbf{R S D}, \mathbf{\%}, \mathbf{n}=\mathbf{6})\end{array}$ & $\begin{array}{c}\text { Stability } \\
(\mathbf{R S D}, \mathbf{\%}, \mathbf{n}=\mathbf{6})\end{array}$ & \multicolumn{2}{c}{ Recovery } \\
\hline & & & & Measured (\%) & RSD (\%) \\
1 & N-R1 & 1.78 & 3.12 & 4.43 & 99.25 & 3.45 \\
2 & G-Rg1 & 3.23 & 4.93 & 3.93 & 101.21 & 2.45 \\
3 & G-Re & 3.65 & 2.86 & 4.43 & 103.19 & 0.94 \\
4 & G-Rf & 4.87 & 4.05 & 4.60 & 99.92 & 2.74 \\
5 & G-F3 & 4.52 & 4.86 & 3.53 & 100.60 & 3.22 \\
6 & G-Rg2 & 1.32 & 4.19 & 3.79 & 101.40 & 1.90 \\
7 & G-Rh1 & 4.72 & 4.11 & 2.09 & 100.22 & 2.68 \\
8 & G-Rb1 & 4.59 & 4.10 & 2.02 & 104.10 & 0.69 \\
9 & G-Ro & 2.99 & 1.40 & 1.02 & 100.38 & 2.84 \\
10 & G-Rc & 4.16 & 2.90 & 2.60 & 102.00 & 2.37 \\
11 & G-Rb2 & 3.78 & 3.70 & 4.43 & 102.29 & 2.61 \\
12 & G-Rb3 & 2.29 & 4.26 & 4.56 & 102.67 & 1.56 \\
13 & GS-IV & 2.70 & 4.32 & 2.47 & 100.98 & 2.68 \\
14 & GS-IVa & 3.18 & 4.74 & 1.67 & 101.55 & 1.89 \\
15 & G-Rd & 4.21 & 3.34 & 3.72 & 100.73 & 3.14 \\
16 & G-Rg3 & 2.72 & 3.41 & 3.31 & 101.67 & 2.77 \\
\hline
\end{tabular}




\subsection{Determination of the 16 Ginsenosides Using HPLC-ESI-MS/MS}

The contents of the major 16 ginsenosides from 50 batch samples were determined using the HPLC-ESI-MS/MS method, including 6 PPDs (G-Rb1, Rb2, Rb3, Rc, Rd, and Rg3), 7 PPTs (G-Re, Rf, Rg1, Rg2, Rh1, F3, and N-R1), and 3 OAs (G-Ro, CS-IV, and CS-IVa). The chromatograms obtained with reference substances and sample solutions are shown in Figure 4. The determination results are shown in Table 6 and Figure 5. In PJ, PM, and PZ, the contents of the PPDs were $4.449 \pm 2.902 \%$, $10.793 \pm 6.135 \%$, and $12.607 \pm 4.247 \%$, respectively. Among them, G-Ro, CS-IV, and CS-IVA have the highest content in plants, which is about 20-70 times that of other ginsenosides. Moreover, the content of G-Rb2, G-Rb3, G-RC, G-R3, and NG-R1 in plants is generally low, and some plants are almost undetectable. By analyzing the amount of compound, we can find that the difference in the content of these compounds (G-Rd, G-Rf, G-F3, G-Ro, and CS-IV) is larger than the others between PM and PJ. The differences in the content of NG-R1, G-Rb1, G-F3, G-Rh1, G-Re, G-Rg1, are larger than the others between PM and PZ. The differences in the content of G-Rh1, NG-R1, G-Rg1, G-F3, G-Ro, and CS-IVA between PJ and PZ are significant. In general, PJ and PM are similar, and PZ differs greatly from them.
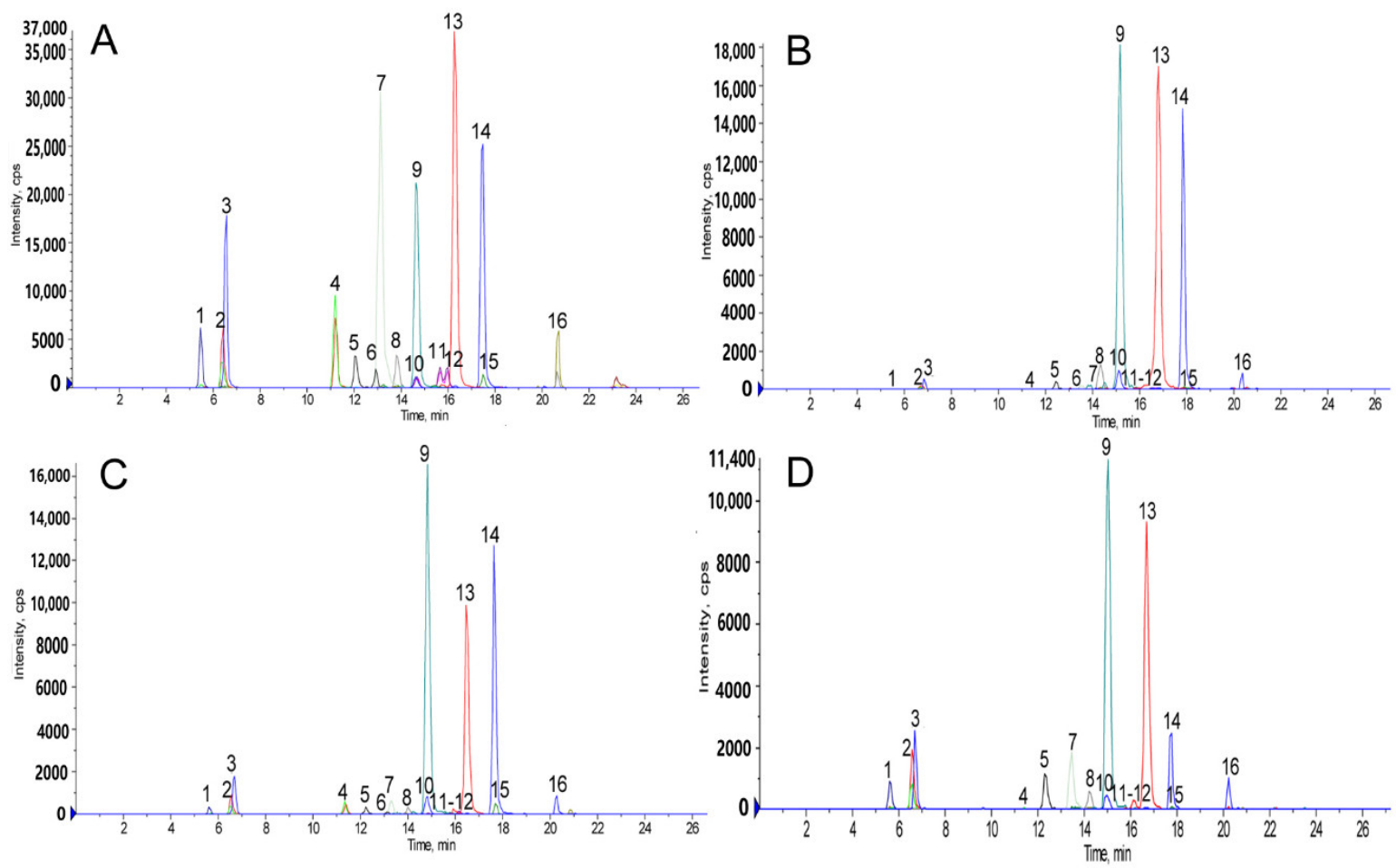

Figure 4. The total ion MRM chromatograms of the reference standards (A) and the roots and rhizomes of Panax japonicas (B), Panax japonicus var. major (C), and Panax zingiberensis (D) obtained in negative mode by HPLC-ESI-MS/MS.

The contents of PPTs were $6.091 \pm 2.143 \%, 6.531 \pm 2.544 \%$, and $52.473 \pm 7.064 \%$. The contents of OAs were $184.357 \pm 42.448 \%, 138.861 \pm 20.353 \%$, and $132.469 \pm 17.248 \%$. The contents of total ginsenosides were $194.897 \pm 44.410 \%, 156.185 \pm 19.814 \%$, and $197.548 \pm 27.227 \%$, respectively. Notably, PZ has a higher level of PPTs content than PJ and PM (by more than 8 times). In addition, G-Ro, CS-IV, and CS-IVa were the three major ginsenosides in PJ and PM, whereas G-Ro, CS-IV, and G-Rg1 were the three major ginsenosides in PZ. Different types of ginsenosides possessed different pharmacological activities. Based on the results, all three drugs clearly contained a large number of OAs and a small amount of dammarane ginsenosides, especially PJ and PM. Furthermore, the content of ginsenosides differed greatly between the three drugs, which may be the reason for their differences in clinical application. 
Table 6. The contents $\left(\mathrm{mg} \times \mathrm{g}^{-1}\right)$ of the 16 ginsenosides in the rhizomes of Panax japonicas (PJ), Panax japonicus var. major (PM), and Panax zingiberensis (PZ).

\begin{tabular}{|c|c|c|c|c|c|c|c|c|c|c|c|c|c|c|c|c|c|c|c|c|}
\hline \multirow{2}{*}{ Batch } & \multicolumn{6}{|c|}{ PPD-Type Ginsenoside } & \multicolumn{7}{|c|}{ PPT-Type Ginsenoside } & \multicolumn{3}{|c|}{ OA-Type Ginsenoside } & \multirow{2}{*}{$\mathrm{T}_{\mathrm{PPD}}$} & \multirow{2}{*}{$\mathrm{T}_{\mathrm{PPT}}$} & \multirow{2}{*}{$\mathrm{T}_{\mathrm{OA}}$} & \multirow{2}{*}{$\mathrm{T}$} \\
\hline & $G-R b_{1}$ & G-Rb ${ }_{2}$ & G-Rb $b_{3}$ & G-Rc & G-Rd & G-Rg 3 & NG-R ${ }_{1}$ & G-Rg 1 & G-Re & G-Rf & G-F $F_{3}$ & G-Rg 2 & $\mathrm{G}-\mathrm{Rh}_{1}$ & G-Ro & CS-IV & CS-IVa & & & & \\
\hline PM-1 & 5.104 & 0.240 & 0.221 & 0.037 & 2.320 & 0.053 & 0.039 & 1.888 & 3.328 & 2.816 & 2.480 & 1.424 & 0.298 & 55.200 & 46.240 & 29.760 & 7.976 & 12.273 & 131.200 & 151.448 \\
\hline PM-2 & 5.664 & 0.266 & 0.245 & 0.011 & 1.344 & 0.026 & 0.057 & 2.064 & 2.864 & 2.352 & 1.558 & 1.195 & 0.381 & 50.880 & 45.760 & 31.680 & 7.555 & 10.472 & 128.320 & 146.346 \\
\hline PM-3 & 4.016 & 0.149 & 0.160 & 0.016 & 0.958 & 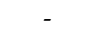 & 0.041 & 0.786 & 1.984 & 1.744 & 1.302 & 0.925 & 0.159 & 53.920 & 49.440 & 31.584 & 5.300 & 6.941 & 134.944 & 147.185 \\
\hline PM-4 & 3.648 & 0.113 & 0.173 & 0.009 & 0.960 & 0.026 & 0.039 & 1.197 & 1.616 & 1.840 & 1.416 & 1.003 & 0.181 & 63.680 & 44.640 & 32.000 & 4.929 & 7.292 & 140.320 & 152.541 \\
\hline PM-5 & 5.488 & 0.246 & 0.259 & 0.042 & 1.184 & 0.054 & 0.025 & 1.022 & 2.464 & 2.096 & 1.824 & 1.158 & 0.151 & 61.760 & 41.120 & 43.040 & 7.273 & 8.740 & 145.920 & 161.934 \\
\hline PM-6 & 3.472 & 0.278 & 0.275 & 0.014 & 0.699 & 0.027 & 0.024 & 1.616 & 1.541 & 1.595 & 1.453 & 0.738 & 0.221 & 71.040 & 49.120 & 34.560 & 4.766 & 7.188 & 154.720 & 166.673 \\
\hline PM-7 & 2.944 & 0.109 & 0.158 & 0.009 & 0.765 & 0.056 & 0.089 & 1.726 & 2.080 & 1.515 & 1.320 & 0.483 & 0.162 & 82.080 & 27.520 & 40.960 & 4.040 & 7.375 & 150.560 & 161.975 \\
\hline PM-8 & 5.712 & 0.139 & 0.182 & 0.005 & 0.890 & 0.026 & 0.056 & 1.507 & 3.008 & 1.525 & 1.808 & 0.576 & 0.376 & 106.080 & 19.840 & 60.160 & 6.955 & 8.856 & 186.080 & 201.891 \\
\hline PM-9 & 3.232 & 0.080 & 0.118 & 0.008 & 0.539 & - & 0.078 & 1.352 & 4.096 & 1.603 & 2.624 & 0.392 & 0.317 & 104.800 & 27.840 & 54.240 & 3.977 & 10.462 & 186.880 & 201.319 \\
\hline PM-10 & 3.904 & 0.200 & 0.296 & - & 14.557 & - & 0.259 & 0.059 & 0.136 & 0.666 & 2.656 & 0.099 & 0.111 & 78.560 & 23.856 & 29.120 & 18.957 & 3.986 & 131.536 & 154.479 \\
\hline PM-11 & 4.256 & 0.250 & 0.231 & - & 13.240 & 0.067 & 0.291 & 0.026 & 0.166 & 0.518 & 2.688 & 0.013 & 0.130 & 81.600 & 22.338 & 33.440 & 18.044 & 3.832 & 137.378 & 159.253 \\
\hline PM-12 & 4.240 & 0.274 & 0.489 & - & 17.056 & 0.053 & 0.347 & - & 0.099 & 0.678 & 2.726 & 0.009 & 0.130 & 76.160 & 27.040 & 28.640 & 22.112 & 3.991 & 131.840 & 157.943 \\
\hline PM-13 & 4.736 & 0.168 & 0.386 & - & 15.824 & 0.026 & 0.293 & - & 0.163 & 0.929 & 3.086 & 0.058 & - & 78.880 & 27.200 & 26.400 & 21.140 & 4.530 & 132.480 & 158.150 \\
\hline PM-14 & 4.992 & 0.355 & 0.292 & - & 15.744 & 0.111 & 0.307 & - & 0.077 & 0.747 & 2.720 & 0.035 & - & 75.520 & 27.360 & 33.440 & 21.494 & 3.886 & 136.320 & 161.701 \\
\hline PM-15 & 3.296 & 0.255 & 0.296 & - & 5.894 & - & 0.235 & 0.421 & 0.714 & 0.208 & 3.936 & 0.451 & 0.062 & 91.200 & 11.360 & 35.680 & 9.741 & 6.027 & 138.240 & 154.008 \\
\hline PM-16 & 2.992 & 0.267 & & - & 5.304 & - & 0.232 & 0.200 & 0.904 & 0.176 & 4.443 & 0.291 & 0.117 & 96.160 & 12.960 & 40.320 & 8.812 & 6.364 & 149.440 & 164.617 \\
\hline PM-17 & 2.464 & 0.330 & 0.354 & - & 7.968 & 0.040 & 0.206 & 0.331 & 0.387 & 0.136 & 3.376 & 0.205 & 0.090 & 65.120 & 6.128 & 46. & 11.155 & 4.732 & 117.328 & 133.215 \\
\hline PM-18 & 3.024 & 0.485 & 0.376 & - & 6.640 & 0.026 & 0.234 & 0.232 & 0.429 & 0.126 & 2.576 & 0.302 & 0.102 & 81.600 & 6.544 & 37.760 & 10.551 & 4.001 & 125.904 & 140.456 \\
\hline PM-19 & 1.984 & 0.354 & 0.22 & - & 6.848 & 0.036 & 0.261 & 0.378 & 0.338 & 0.068 & 3.504 & 0.192 & 0.10 & 75.840 & 5.216 & 30.880 & 9.445 & 4.847 & 111.936 & 126.228 \\
\hline PM-20 & 2.640 & 0.461 & 0.635 & - & 7.904 & - & 0.278 & 0.224 & 0.208 & 0.099 & 3.632 & 0.300 & 0.089 & 66.880 & 4.272 & 34 & 11.640 & 4.830 & 105.872 & 122.342 \\
\hline Mean & 3.890 & 0.251 & 0.281 & 0.008 & 6.332 & 0.031 & 0.170 & 0.751 & 1.330 & 1.072 & 2.556 & 0.493 & 0.159 & 75.848 & 26.290 & 36.723 & 10.793 & 6.531 & 138.861 & 156.185 \\
\hline $\mathrm{SD}(\mathrm{n}=20)$ & 1.110 & 0.110 & 0.122 & 0.012 & 5.925 & 0.029 & & 0.722 & & 0.851 & 0.926 & 0.437 & 0.109 & 15.590 & & & & & & 19.814 \\
\hline PJ-1 & 1.626 & 0.061 & - & - & 0.355 & 0.031 & 0.055 & 5.360 & 2.016 & 0.422 & 0.418 & 0.136 & - & 88.480 & 50.720 & 10.880 & 2.07 & 8.407 & 150.080 & 160.560 \\
\hline PJ-2 & 1.280 & - & - & - & 0.352 & 0.029 & - & 3.232 & 2.432 & 0.650 & 0.789 & 0.189 & 0.130 & 101.120 & 37.440 & 10.544 & & 7.422 & 149.104 & 158.186 \\
\hline PJ-3 & 1.363 & - & - & 0.010 & 0.432 & 0.054 & - & 2.128 & 1.427 & 1.099 & 0.571 & 0.131 & 0.130 & 70.080 & 39.680 & 8.816 & 1.8 & 5.487 & 118.576 & 125.922 \\
\hline PJ-4 & 1.280 & 0.009 & - & - & 0.917 & 0.004 & 0.010 & 2.992 & 1.760 & 0.512 & 0.922 & 0.158 & - & 77.280 & 33.600 & - & 2.2 & 6.354 & 120.880 & 129.443 \\
\hline PJ-5 & 1.499 & & 0.028 & - & 0.701 & 0.056 & & 4.560 & 1.760 & 0.6 & 0.944 & & - & 77.600 & & & & 8.039 & & 125.746 \\
\hline PJ-6 & 7.728 & 0.406 & 0.5 & - & 1.648 & - & 0.034 & 0.669 & 1.619 & 0.020 & 2.6 & 0.27 & - & 141.280 & 49.280 & 54.880 & 10.331 & 5.257 & 245.440 & 261.028 \\
\hline PJ-7 & 9.232 & 0.219 & & - & 1.363 & 0.041 & 0.036 & & 2.3 & & 2.7 & & - & & 45.920 & & & 6.366 & 240.160 & 257.798 \\
\hline PJ-8 & 2.064 & 0.328 & & - & 1.624 & & & 0.294 & 1.274 & 0.0 & 2.896 & 0.4 & - & 99.520 & 48.160 & 46.400 & 4.400 & 5.078 & 194.080 & 203.558 \\
\hline PJ-9 & & 0.674 & & - & & & & & & & & & - & & & & & 5.750 & & 204.044 \\
\hline PJ-10 & 2.064 & 0.248 & 0.2 & 0.099 & 1.574 & & & 2.19 & & & 1.8 & & 0.162 & & 39.360 & & & 6.068 & & 176.587 \\
\hline PJ-11 & 5.456 & & & 0.054 & 1.512 & & & 1.68 & 0.7 & 0. & 2.3 & 0.1 & 0.115 & 118 & 41.600 & 44 & 7.6 & 5.222 & 205.120 & 217.984 \\
\hline PJ-12 & 5.792 & 0.707 & 0.3 & 0.038 & 2.256 & 0.02 & & 0.072 & 0.0 & 0.011 & 1.640 & 0.164 & 0.091 & 145.600 & 34.240 & 69.600 & 9.140 & 2.079 & 249.440 & 260.658 \\
\hline PJ-13 & 1.114 & 0.126 & & 0.300 & 0.187 & & & 3.904 & & 0.302 & 0.334 & 0.0 & 0.584 & 137.920 & 57.280 & 16. & 1.926 & 8.270 & 211.360 & 221.556 \\
\hline PJ-14 & & & & 0.250 & & & & 2.68 & & & 0.3 & 0.0 & & 145 & 62. & & 2.2 & 7.816 & 222.416 & 232.503 \\
\hline PJ-15 & 2.112 & 0.215 & 0.117 & 0.277 & 0.514 & & & 4.032 & & 0.2 & 0.475 & 0.1 & 0.290 & & & & 3.260 & 9.151 & 216.608 & 229.019 \\
\hline PJ-16 & 1.595 & & & 0.328 & & & & 4.624 & & 0.2 & 0.627 & 0.1 & 0.290 & & 65.440 & & 3.183 & 10.159 & 226.528 & 239.870 \\
\hline PJ-17 & 3.146 & 0.434 & 0.248 & 0.171 & 1.216 & 0.026 & 0.121 & 0.469 & 1.309 & 0.031 & 1.376 & 0.656 & - & 74.080 & 39.360 & 54.720 & 5.240 & 3.962 & 168.160 & 177.363 \\
\hline PJ-18 & 2.496 & & & - & 1.080 & 0.041 & & 0.298 & 1.062 & & 0.869 & & - & 76.960 & 46.080 & & 4.061 & 2.823 & 177.600 & 184.484 \\
\hline PJ-19 & 2.269 & 0.166 & 0.181 & 0.018 & 0.981 & - & & 0.290 & 2.224 & & 0.832 & 0.4 & - & 78.080 & 47.040 & & 3.614 & 3.951 & 161.600 & 169.166 \\
\hline PJ-20 & 1.680 & 0.326 & 0.202 & 0.037 & 1.152 & 0.028 & 0.234 & 0.989 & 0.7 & 0.0 & 1.424 & 0.5 & 0.130 & 85.760 & 37.280 & & 3.425 & 4.156 & 154.880 & 162.461 \\
\hline Mean & 2.860 & & & 0.079 & 1.024 & & & 2.087 & 1.957 & & 1.340 & 0.271 & 0.109 & 106.680 & 46.272 & & 4.449 & 6.091 & 184.357 & 194.897 \\
\hline $\mathrm{SD}(\mathrm{n}=20)$ & 2.310 & 0.203 & 0.168 & 0.116 & 0.597 & 0.022 & 0.069 & 1.730 & 1.093 & 0.299 & 0.898 & 0.173 & 0.152 & 29.528 & 10.162 & 20.349 & 2.902 & 2.143 & 42.448 & 44.410 \\
\hline
\end{tabular}


Table 6. Cont.

\begin{tabular}{|c|c|c|c|c|c|c|c|c|c|c|c|c|c|c|c|c|c|c|c|c|}
\hline \multirow{2}{*}{ Batch } & \multicolumn{6}{|c|}{ PPD-Type Ginsenoside } & \multicolumn{7}{|c|}{ PPT-Type Ginsenoside } & \multicolumn{3}{|c|}{ OA-Type Ginsenoside } & \multirow{2}{*}{$\mathrm{T}_{\mathrm{PPD}}$} & \multirow{2}{*}{$\mathrm{T}_{\mathrm{PPT}}$} & \multirow{2}{*}{$\mathrm{T}_{\mathrm{OA}}$} & \multirow{2}{*}{$\mathrm{T}$} \\
\hline & $\mathrm{G}-\mathbf{R} \mathbf{b}_{1}$ & $\mathrm{G}-\mathbf{R b}_{2}$ & $\mathrm{G}-\mathrm{Rb}_{3}$ & G-Rc & G-Rd & G-Rg $g_{3}$ & NG-R ${ }_{1}$ & G-Rg 1 & G-Re & G-Rf & $\mathrm{G}-\mathrm{F}_{3}$ & G-Rg 2 & $\mathrm{G}-\mathrm{Rh}_{1}$ & G-Ro & CS-IV & CS-IVa & & & & \\
\hline PZ-1 & 9.136 & 0.178 & 0.029 & 0.014 & 0.450 & 0.450 & 3.488 & 24.640 & 10.960 & 0.058 & 10.080 & 2.912 & 3.984 & 93.760 & 25.600 & 11.472 & 10.256 & 56.122 & 130.832 & 197.209 \\
\hline PZ-2 & 8.128 & & & & & & & 21.760 & 10.128 & 0.079 & 10.112 & 2.992 & 2.880 & 91.520 & 25.760 & & 9.146 & 1.711 & & 189.082 \\
\hline PZ-3 & 8.464 & & & & & & & 20.320 & 8.768 & 0.080 & 9.392 & 2.944 & & 86.080 & 25.120 & 10.560 & 9.324 & 8.048 & 121.760 & 179.133 \\
\hline PZ-4 & 8.256 & 0.02 & 0.05 & 0.020 & & 0.3 & 3.888 & 17.040 & 8.736 & 0.050 & 9.712 & 3.376 & 2.160 & 80.960 & 23.840 & 9.744 & 9.244 & 4.962 & 114.544 & 168.750 \\
\hline PZ-5 & 8.288 & & & & & & & & 9.440 & & 11.552 & 3.440 & & 84.160 & & & 9.190 & 46.227 & & 175.641 \\
\hline PZ-6 & & & & & & & & & & & 10.480 & & & & & & & & & 183.768 \\
\hline PZ-7 & .528 & & & 0 & 0.374 & & & 22.085 & 14.720 & 0.056 & 5.5 & 0.8 & 5.1 & 99.680 & 22.080 & 11.7 & 13.048 & 52.208 & 133.552 & 198.808 \\
\hline PZ-8 & & & & & & & & 22.402 & 15.808 & & 4.7 & & & & & & & & & 193.629 \\
\hline PZ-9 & 5.840 & 0.408 & 0.397 & & 0.35 & & 5.4 & 22.254 & 15.344 & 0.0 & 10.5 & 2.1 & & 118.080 & 28.000 & & 17.447 & 43 & 155.952 & 232.642 \\
\hline PZ-10 & & & & & & & & 26.244 & 16.960 & 0.0 & 12.6 & 2.352 & 3.9 & 125.600 & 29.440 & 14.8 & 18.930 & 68.036 & 169.856 & 256.821 \\
\hline Mean & 10.978 & 0.369 & 0.327 & 0.059 & 0.522 & 0.352 & 4.219 & 20.907 & 12.024 & 0.066 & 9.490 & 2.593 & 3.17 & 95.840 & 24.752 & 11.877 & 12.607 & 52.473 & 132.469 & 197.548 \\
\hline $\mathrm{SD}(\mathrm{n}=10)$ & 3.510 & 0.408 & 0.427 & 0.068 & 0.234 & 0.084 & 0.817 & 3.479 & 3.280 & 0.020 & 2.476 & 0.859 & 1.005 & 14.750 & 3.097 & 1.848 & 4.247 & 7.064 & 17.248 & 27.227 \\
\hline
\end{tabular}

$\mathrm{T}_{\mathrm{PPD}}, \mathrm{T}_{\mathrm{PPT}}, \mathrm{T}_{\mathrm{OA}}$, and $\mathrm{T}$ represent the content of protopanaxdiol-type ginsenosides, protopanaxtriol-type ginsenosides, oleanolic acid-type ginsenosides, and total ginsenosides, respectively;

"-" represents not detected; $\mathrm{SD}$, standard deviation. 


\section{A}

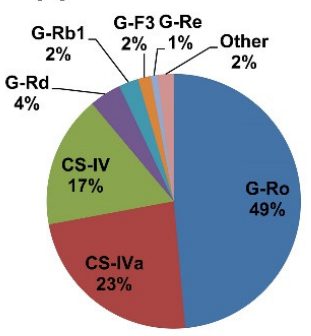

C

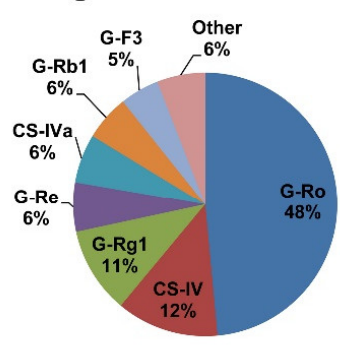

B

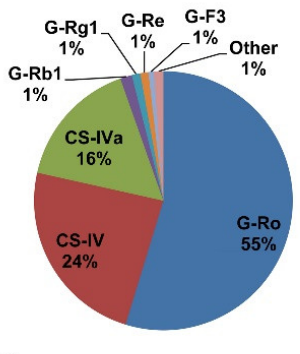

D

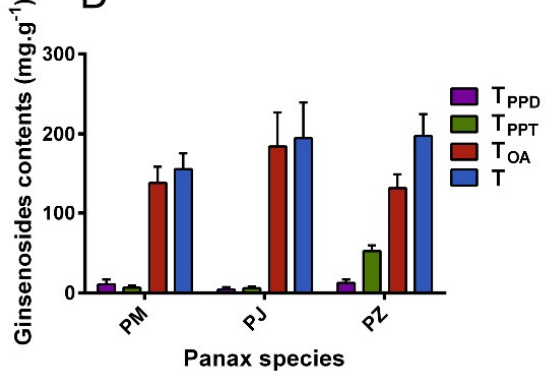

E

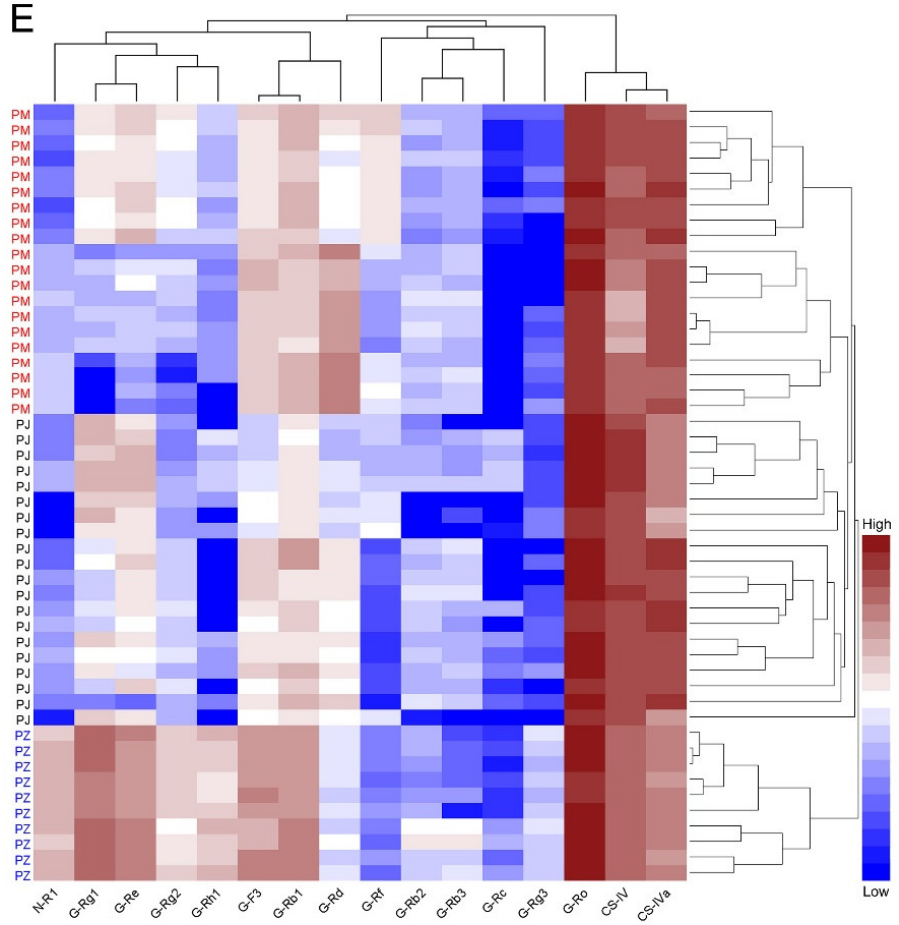

Figure 5. The percentage contents of the 16 ginsenosides (A, PJ; B, PM; C, PZ) and total ginsenosides (D) in the three ginseng herbs; The content difference of 16 saponins by a two-way hierarchical clustering analysis heat map (E). Ginsenoside (G)-Rg1, G-Re, G-Rb1, G-Rc, G-Rb2, G-Rb3, G-Rf, G-Rd, G-Rg2, G-Rg3, G-F3, G-Rh1, G-Ro. Notoginsenoside (N)-R1 and chikusetsu saponin (CS) -IV and CS-Iva.

\subsection{Discrimination of PJ, PM, and PZ by a Multivariate Statistical Analysis}

The variations in the 16 major compounds among the three ginseng species were intuitively represented by a two-way hierarchical clustering analysis heat map. As shown in the heat map in Figure 5E, PZ could be clearly distinguished from PJ and PM by a hierarchical clustering analysis. In contrast, PJ and PZ were not well-discriminated, and their ginsenoside contents were much closer than that of PZ. 
In order to further reveal differences in the chemical composition among PJ, PM, and PZ, PLS-DA and OPLS-DA were also utilized to distinguish between different ginseng species. A total of 50 ginseng samples (20 batches of PJ; 20 batches of PM; 10 batches of PZ) were analyzed. Among them, 35 samples were randomly selected as the training set, and 15 were the prediction set. The result is shown in Figure 6. The established PLS-DA model showed good fitness (R2X =0.699, R2Y $=0.897$ ) and predictability $(\mathrm{Q} 2=0.85)$. The PLS-DA score plot displayed that the three clusters representing the PJ, PM, and PZ groups were well-segregated, thereby indicating the remarkable differences of ginsenosides among these three Panax herbs. A chance permutation test suggested that the model was not over-fitted (as shown in Figure 6A-3). All samples in the prediction set are correctly identified, so the PLS-DA model has a classification accuracy of $100 \%$. The VIP (variable importance in the projection) plot was used to find the important compounds. When the VIP cutoff was set at 1.0, nine important compounds for discriminating between PJ, PM, and PZ were discovered. G-Rf, G-F3, and CS-IV have the highest contribution that can distinguish the above three herbs (as shown in Figure 6A-4).

The result of the OPLS-DA analysis based on pairwise comparison methods is shown in Figure 6B-D. The figure reveals that samples from the same species were tightly clustered together and that different species groups were discriminated from one another by the OPLS-DA score plot. Among those with a VIP value exceeding 1.0, seven important compounds for the discrimination between PJ and PM (in order of CS-IV, G-Rf, G-F3, G-Rd, G-Ro, G-Rb1, and G-Rg1), nine for PM and PZ (in order of G-Rh1, N-R1, G-F3, G-Rb1, CS-IVa, G-Rg1, G-Re, G-Rg3, and G-Rf), and nine for PJ and PZ (in order of G-Rh1, N-R1, G-Rg2, G-F3, G-Rg3, G-Rg1, G-Rb1, G-Re, and CS-IV) were obtained.

In Chinese folk medicine, PJ and PM are historically and generally used as a herbal medicine for similar indications. These two herbs possess combined medicinal effects, which are $P$. ginseng's "conserving vitality" activities and P. notoginseng's "replenishing blood" activities [10]. However, PJ and PM are considered to be two different herbal medicines, and are recorded as "zhujieshen" and "zhuzishen" in the Chinese Pharmacopoeia, respectively. Thus, investigation on the difference in their chemical constituents and biopharmalogical effects is necessary and crucial. In our qualitative and quantitative analyses based on an LC-MS technique, PLS-DA and OPLS-DA clearly distinguished between the two herbs, even though they are very similar in composition. Furthermore, the average content of total ginsenosides in PJ was conspicuously higher than that in PM, even though PM's content in PPDs and PPTs was more abundant. These chemical differences may contribute to the differences in clinical application.

$\mathrm{PZ}$, commonly known as ginger ginseng or Myanmar ginseng, is indigenous to Yunnan province in the Southwest of China [13], and has also been reported to wildly grow at the Par Moe Ne Water Spring area in Taung-gyi, Shan State, Myanmar, which has an altitude of $1500 \mathrm{~m}$ above sea level. Few previous studies have focused on the comprehensive chemical compositions and determination of ginsenosides in PZ [26]. In the present study, for the first time, the chemical compositions of this herb were screened and identified by UHPLC-Q-Exactive/HRMS, and the content of 16 major ginsenosides was determined. These results are beneficial for the development and quality assessment of PZ.

In conclusion, an integrated strategy to comprehensively identify the chemical composition and simultaneously quantify 16 ginsenodsies in the three Panax herbs was successfully established. After optimization of the conditions, a total of 101 ginsenosides were detected and tentatively identified using UHPLC-Q-Exactive/HRMS, including 82 from PJ, 78 from PM, and 67 from PZ. Among these compounds, 22 were unambiguously confirmed by comparing their retention times and mass spectra with those of reference ginsenosides. The quantitative analysis was implemented using a reliable and practical HPLC-ESI-MS/MS method using MRM mode. The validation of the methodology showed favorable levels of LOD, LOQ, linearity, precision, repeatability, stability, and recovery. Finally, the PLS-DA and OPLS-DA results, based on the quantitative data, displayed a significant difference in ginsenoside content between the three ginseng-drugs. G-Rf, G-F3, and CS-IV were the three most characteristic components that can distinguish between the three herbs. The integrated LC-MS-based 
strategy combined with a multivariate data analysis could not only achieve a rapid, accurate, and comprehensive qualitative and quantitative analysis of the complex ginsenoside but also enable us to discriminate between $\mathrm{PJ}, \mathrm{PM}$, and PZ, which provides valuable references for the quality assessment and control of traditional Chinese medicines (TCMs).
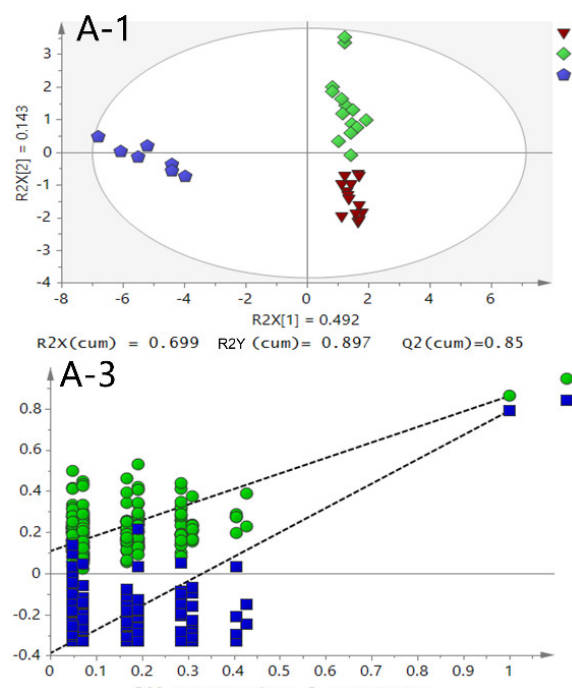

200 permutations 3 components
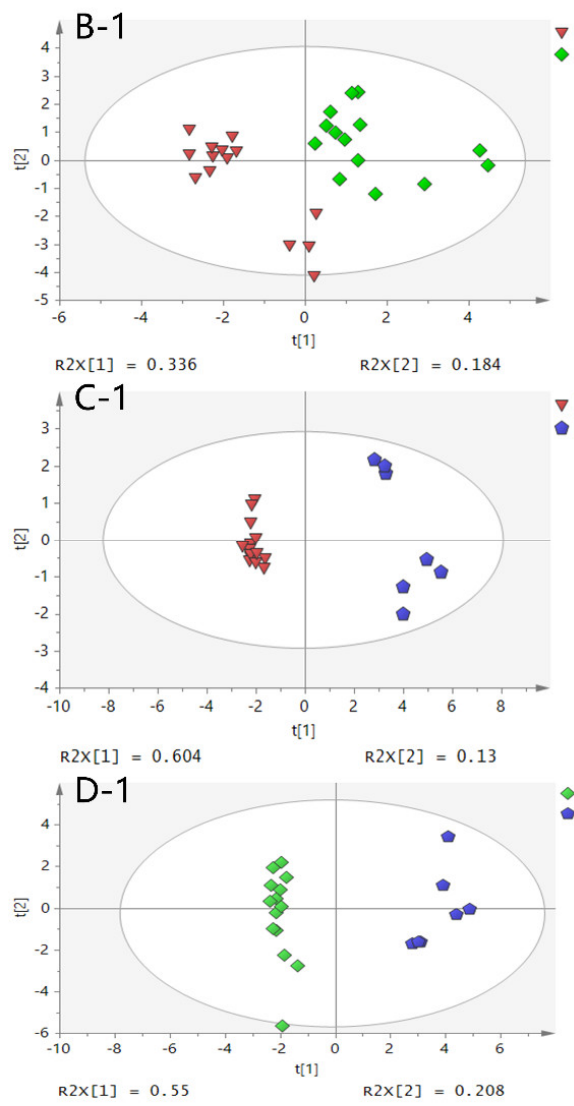

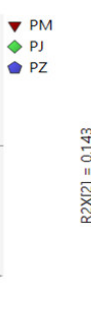

- $\mathrm{Q} 2$
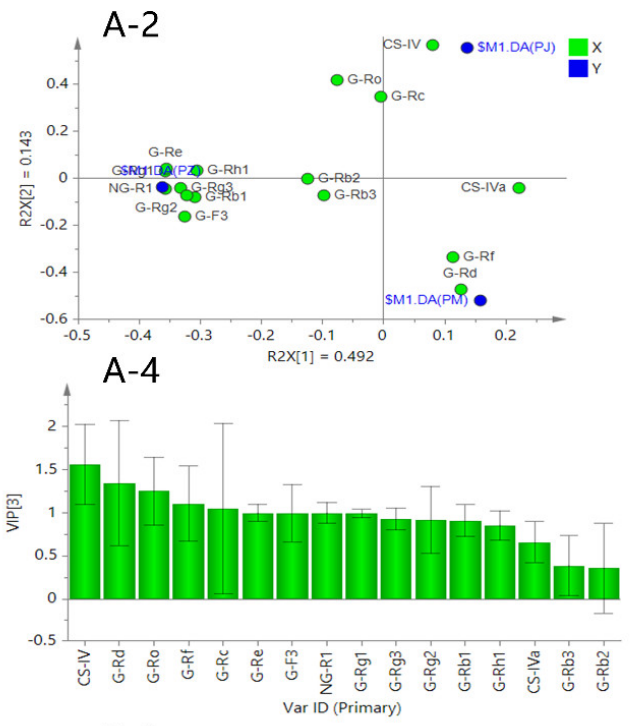

B-2

P

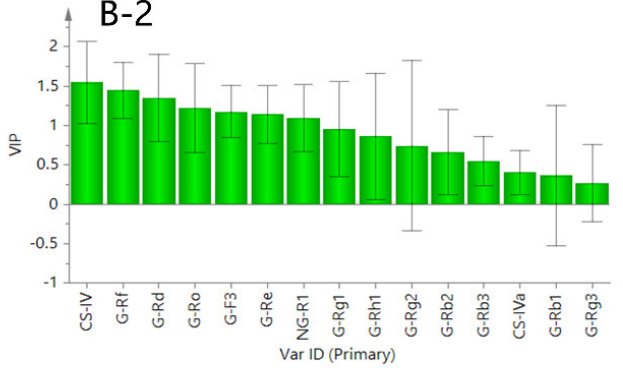

C-2

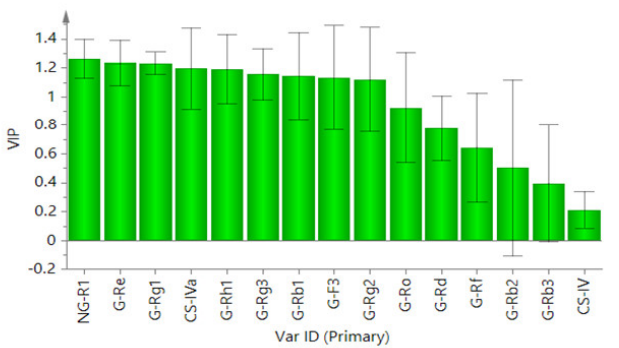

PI

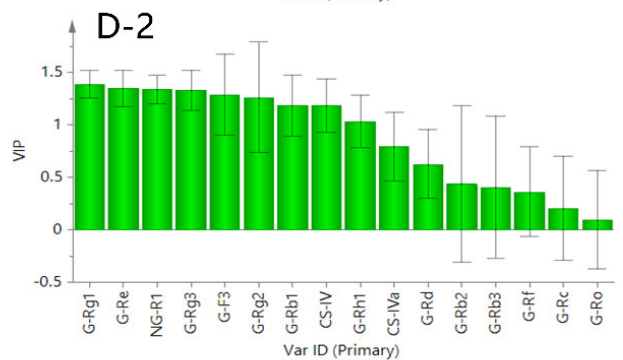

Figure 6. The PLS-DA (partial least squares-discriminate analysis) score scatter plot (A-1), the loading scatter plot (A-2), the chance permutation test to validate the occurrence or absence of over-fitting of the PLS-DA (partial least squares-discriminate analysis) model (A-3), the VIP (variable importance in the projection) values of the 16 saponins (A-4), the OPLS-DA (orthogonal partial least squares-discriminant analysis) score scatter plot based on a pairwise comparison (B-1, C-1, D-1), and the VIP values of the 16 saponins (B-2, C-2, D-2). 
Author Contributions: Z.D. and J.L. designed the study. Z.D., J.L., X.Z. processed the data, performed the analyses and analyzed the results, and wrote the manuscript. J.P., L.H., Z.D., J.L., X.Z. edited the manuscript. All authors read and approved the final version of the manuscript.

Funding: The work was supported by grants from the National Natural Science Foundation of China (No. 81274013, No. 81473315), the CAMS Innovation Fund for Medical Sciences (CIFMS) (No. 2016-I2M-3-015), the Key Projects in the National Science and Technology Pillar Program (NO. 2011BAI07B08).

Conflicts of Interest: The authors declare no conflict of interest.

\section{References}

1. Qi, L.W.; Wang, C.Z.; Yuan, C.S. Isolation and analysis of ginseng: advances and challenges. Nat. Prod. Rep. 2011, 28, 467-495. [CrossRef] [PubMed]

2. Baeg, I.H.; So, S.H. The world ginseng market and the ginseng (Korea). J. Ginseng Res. 2013, 37, 1-7. [CrossRef] [PubMed]

3. Xu, W.; Choi, H.K.; Huang, L.J.M. State of Panax ginseng Research: A Global Analysis. Molecules 2017, 22, 1518. [CrossRef] [PubMed]

4. Kim, Y.S.; Woo, J.Y.; Han, C.K.; Chang, I.M. Safety Analysis of Panax Ginseng in Randomized Clinical Trials: A Systematic Review. Medicines 2015, 2, 106-126. [CrossRef] [PubMed]

5. Du, Z.; Wu, J.; Meng, X.; Li, J.; Huang, L. Predicting the Global Potential Distribution of Four Endangered Panax Species in Middle-and Low-Latitude Regions of China by the Geographic Information System for Global Medicinal Plants (GMAPGIS). Molecules 2017, 22, 1630. [CrossRef] [PubMed]

6. Wang, H.-P.; Zhang, Y.-B.; Yang, X.-W.; Zhao, D.-Q.; Wang, Y.-P. Rapid characterization of ginsenosides in the roots and rhizomes of Panax ginseng by UPLC-DAD-QTOF-MS/MS and simultaneous determination of 19 ginsenosides by HPLC-ESI-MS. J. Ginseng Res. 2016, 40, 382-394. [CrossRef] [PubMed]

7. Michalski, A.; Damoc, E.; Hauschild, J.-P.; Lange, O.; Wieghaus, A.; Makarov, A.; Nagaraj, N.; Cox, J.; Mann, M.; Horning, S. Mass spectrometry-based proteomics using Q Exactive, a high-performance benchtop quadrupole Orbitrap mass spectrometer. Mol. Cell Proteomics 2011, 10. [CrossRef] [PubMed]

8. Wang, Q.; Jiang, P.; Ye, F.-Y.; Shi, R.; Ma, Y.-M.; Zhong, J.; Wu, J.-S.; Liu, P.; Liu, C.-H.; Jia, Y.-Q. Identification and pharmacokinetics of multiple constituents in rat plasma after oral administration of Yinchenzhufu decoction. J. Ethnopharmacol. 2014, 153, 714-724. [CrossRef] [PubMed]

9. Li, Q.; Zhao, Y.; Zhu, D.; Pang, X.; Liu, Y.; Frew, R.; Chen, G. Lipidomics profiling of goat milk, soymilk and bovine milk by UPLC-Q-Exactive Orbitrap Mass Spectrometry. Food Chem. 2017, 224, 302-309. [CrossRef] [PubMed]

10. Hu, M.; Müller, E.; Schymanski, E.L.; Ruttkies, C.; Schulze, T.; Brack, W.; Krauss, M. Performance of combined fragmentation and retention prediction for the identification of organic micropollutants by LC-HRMS. Anal. Bioanal. Chem. 2018, 410, 1931-1941. [CrossRef] [PubMed]

11. Cicero, N.; Albergamo, A.; Salvo, A.; Bua, G.D.; Bartolomeo, G.; Mangano, V.; Rotondo, A.; Di Stefano, V.; Di Bella, G.; Dugo, G. Chemical characterization of a variety of cold-pressed gourmet oils available on the Brazilian market. Food Res. Int. 2018, 109, 517-525. [CrossRef] [PubMed]

12. Sato, E.; Saigusa, D.; Mishima, E.; Uchida, T.; Miura, D.; Morikawaichinose, T.; Kisu, K.; Sekimoto, A.; Saito, R.; Oe, Y.; et al. Impact of the Oral Adsorbent AST-120 on Organ-Specific Accumulation of Uremic Toxins: LC-MS/MS and MS Imaging Techniques. Toxins 2018, 10, 19. [CrossRef] [PubMed]

13. Delatour, T.; Racault, L.; Bessaire, T.; Desmarchelier, A. Screening of Veterinary Drug Residues in Food by LC-MS/MS. Background and Challenges. Food Addit. Contam. Part. A Chem. Anal. Control Expo. Risk Assess. 2018, 35, 632-645. [CrossRef] [PubMed]

14. Zhang, Y.; Guo, L.; Duan, L.; Dong, X.; Zhou, P.; Liu, E.H.; Li, P. Simultaneous determination of 16 phenolic constituents in Spatholobi Caulis by high performance liquid chromatography/electrospray ionization triple quadrupole mass spectrometry. J. Pharmaceut. Biomed. 2015, 102, 110-118. [CrossRef] [PubMed]

15. Shi, X.J.; Yang, W.Z.; Qiu, S.; Yao, C.L.; Shen, Y.; Pan, H.Q.; Bi, Q.R.; Yang, M.; Wu, W.Y.; Guo, D.A. An in-source multiple collision-neutral loss filtering based nontargeted metabolomics approach for the comprehensive analysis of malonyl-ginsenosides from Panax ginseng, P. quinquefolius, and P. notoginseng. Anal. Chim. Acta 2017, 952, 59-70. [CrossRef] [PubMed] 
16. Pace, R.; Martinelli, E.M.; Sardone, N.; Combarieu, E.D. Metabolomic evaluation of ginsenosides distribution in Panax genus (Panax ginseng and Panax quinquefolius) using multivariate statistical analysis. Fitoterapia 2015, 101, 80-91. [CrossRef] [PubMed]

17. Yang, X.; Wang, R.; Zhang, S.; Zhu, W.; Tang, J.; Liu, J.; Chen, P.; Zhang, D.; Ye, W.; Zheng, Y. Polysaccharides from Panax japonicus C.A. Meyer and their antioxidant activities. Carbohydr. Polym. 2014, 101, 386-391. [CrossRef] [PubMed]

18. Zhang, S.; Wang, R.; Zeng, W.; Zhu, W.; Zhang, X.; Wu, C.; Song, J.; Zheng, Y.; Chen, P. Resource investigation of traditional medicinal plant Panax japonicus (T.Nees) C.A. Mey and its varieties in China. J. Ethnopharmacol. 2015, 166, 79-85. [CrossRef] [PubMed]

19. He, H.; Xu, J.; Xu, Y.; Zhang, C.; Wang, H.; He, Y.; Wang, T.; Yuan, D. Cardioprotective effects of saponins from Panax japonicus on acute myocardial ischemia against oxidative stress-triggered damage and cardiac cell death in rats. J. Ethnopharmacol. 2012, 140, 73-82. [CrossRef] [PubMed]

20. Liu, Y.; Zhao, J.; Chen, Y.; Li, W.; Li, B.; Jian, Y.; Sabir, G.; Cheng, S.; Tuo, Q.; Khan, I.; et al. Polyacetylenic Oleanane-Type Triterpene Saponins from the Roots of Panax japonicus. J. Nat. Prod. 2016, 79, 3079-3085. [CrossRef] [PubMed]

21. Tran, Q.L.; Than, M.M.; Tezuka, Y.; Banskota, A.H.; Kouda, K.; Watanabe, H.; Zhu, S.; Komatsu, K.; Thet, M.M.; Swe, T.; et al. Wild ginseng grows in Myanmar. Chem. Pharm. Bull. 2003, 51, 679-682. [CrossRef] [PubMed]

22. Yang, L.; Yu, Q.T.; Ge, Y.Z.; Zhang, W.S.; Fan, Y.; Ma, C.W.; Liu, Q.; Qi, L.W. Distinct urine metabolome after Asian ginseng and American ginseng intervention based on GC-MS metabolomics approach. Sci. Rep. 2016, 6, 39045. [CrossRef] [PubMed]

23. Shin, B.K.; Kwon, S.W.; Park, J.H. Chemical diversity of ginseng saponins from Panax ginseng. J. Ginseng Res. 2015, 39, 287-298. [CrossRef] [PubMed]

24. Yang, W.-Z.; Bo, T.; Ji, S.; Qiao, X.; Guo, D.-A.; Ye, M. Rapid chemical profiling of saponins in the flower buds of Panax notoginseng by integrating MCI gel column chromatography and liquid chromatography/mass spectrometry analysis. Food Chem. 2013, 139, 762-769. [CrossRef] [PubMed]

25. Chu, C.; Xu, S.; Li, X.; Yan, J.; Liu, L. Profiling the Ginsenosides of Three Ginseng Products by LC-Q-TOF/MS. J Food Sci. 2013, 78, C653-C659. [CrossRef] [PubMed]

26. Zhu, S.; Zou, K.; Fushimi, H.; Cai, S.; Komatsu, K. Comparative study on triterpene saponins of Ginseng drugs. Planta Med. 2004, 70, 666-677. [CrossRef] [PubMed]

Sample Availability: Samples of the compounds are not available from the authors.

(C) 2018 by the authors. Licensee MDPI, Basel, Switzerland. This article is an open access article distributed under the terms and conditions of the Creative Commons Attribution (CC BY) license (http:// creativecommons.org/licenses/by/4.0/). 